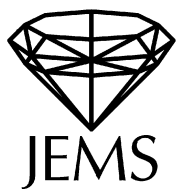

Wei Wang

\title{
Canonical contact forms on spherical CR manifolds
}

\author{
Received March 6, 2002 / final version received January 15, 2003
}

Published online March 3, 2003 - (c) Springer-Verlag \& EMS 2003

\begin{abstract}
We construct the CR invariant canonical contact form can $(J)$ on scalar positive spherical CR manifold $(M, J)$, which is the CR analogue of canonical metric on locally conformally flat manifold constructed by Habermann and Jost. We also construct another canonical contact form on the Kleinian manifold $\Omega(\Gamma) / \Gamma$, where $\Gamma$ is a convex cocompact subgroup of $\operatorname{Aut}_{C R} \mathbf{S}^{2 n+1}=P U(n+1,1)$ and $\Omega(\Gamma)$ is the discontinuity domain of $\Gamma$. This contact form can be used to prove that $\Omega(\Gamma) / \Gamma$ is scalar positive (respectively, scalar negative, or scalar vanishing) if and only if the critical exponent $\delta(\Gamma)<n$ (respectively, $\delta(\Gamma)>n$, or $\delta(\Gamma)=n$ ). This generalizes Nayatani's result for convex cocompact subgroups of $S O(n+1,1)$. We also discuss the connected sum of spherical CR manifolds.
\end{abstract}

\section{Introduction}

The geometry of strictly pseudoconvex CR manifolds has many parallels with Riemannian geometry [BFG], and there is a far reaching analogue between conformal geometry and CR geometry. Jerison and Lee gave a table [JL1, p. 169] summarizing some important parallels. Since a CR diffeomorphism between CR manifolds is a conformal transformation, conformal invariant objects are CR invariant. Thus, the conformal geometry of CR manifolds will contribute to better understanding of CR geometry.

The complex counterparts of locally conformally flat manifolds are spherical $\mathrm{CR}$ manifolds. The purpose of this paper is to construct $\mathrm{CR}$ invariant canonical contact forms on spherical CR manifolds, which are generalizations of HabermannJost's and Nayatani's conformally invariant metrics on locally conformally flat manifolds.

Let $(M, J)$ be a $2 n+1$ dimensional compact and strictly pseudoconvex CR manifold with horizontal subspace $H$. Here $H \subset T M$, $\operatorname{dim} H_{P}=2 n$ for any $P \in M$ and $J$ is a complex structure on $H$. A choice of $1-$ form $\theta$ such that $\operatorname{ker} \theta=H$ is called a pseudohermitian structure on $(M, J)$. Denote by $\{J\}$ the set of strictly pseudoconvex pseudohermitian structures on $(M, J)$. Given a strictly

W. Wang: Department of Mathematics, Zhejiang University (Xixi campus), Zhejiang 310028, P.R. China, e-mail: wangf@mail.hz.zj.cn and Department of Mathematics, University of Toronto, Ontario, Canada M5S 3G3, e-mail: weiwang@math.toronto.edu

Partially supported by National Nature Science Foundation (No. 10071070) in China 
pseudoconvex pseudohermitian structure $\theta$ on a CR manifold, Webster [W] has defined the connection, curvature and scalar curvature associated with $\theta$. We say $\theta$ is conformal to $\theta_{0}$ if $\theta=\phi^{\frac{4}{Q-2}} \theta_{0}$, for some positive smooth function $\phi$ on $M$, where $Q=2 n+2$ is the homogeneous dimension of $M$. A mapping between pseudohermitian manifolds, $f:\left(M_{0}, \theta_{0}\right) \longrightarrow\left(M_{1}, \theta_{1}\right)$, is called conformal if $f^{*} \theta_{1}=\phi^{\frac{4}{Q-2}} \theta_{0}$ for some positive smooth function $\phi$ on $M_{0}$.

We can define the SubLaplacian $\triangle_{\theta}$ associated with $\theta$ in an analogous way to define the Laplacian associated with a Riemannian metric. Let $R_{\theta}$ be the Webster scalar curvature of $\theta$. The conformal SubLaplacian $L_{\theta}=b_{n} \triangle_{\theta}+R_{\theta}, b_{n}=2+\frac{2}{n}$, satisfies the transformation law

$$
L_{\theta} u=\phi^{-\frac{Q+2}{Q-2}} L_{\theta_{0}}(u \phi)
$$

if $\theta=\phi^{\frac{4}{Q-2}} \theta_{0}$ and $u \in C^{\infty}(M)$. If we put $u=1$, then we obtain the transformation law of the Webster scalar curvatures

$$
R_{\theta}=\phi^{-\frac{Q+2}{Q-2}}\left(b_{n} \triangle_{\theta_{0}}+R_{\theta_{0}}\right)(\phi) .
$$

Thus, $\phi$ satisfies the scalar curvature equation

$$
b_{n} \triangle_{\theta_{0}} \phi+R_{\theta_{0}} \phi=R_{\theta} \phi^{\frac{Q+2}{Q-2}} .
$$

A CR manifold $(M, J)$ is called spherical if it is locally CR equivalent to an open set of the sphere $\mathbf{S}^{2 n+1}$ with the standard complex structure, where $2 n+1=\operatorname{dim} M$. We can show that there is one and only one of the following cases holding: $\{J\}$ contains a contact form with either (1) positive, or (2) negative, or (3) vanishing Webster scalar curvature. So, we can call a CR structure either scalar positive, or scalar negative, or scalar vanishing, respectively.

Theorem 1.1. Let $(M, J)$ be a connected, compact, scalar positive, spherical $C R$ manifold with $\operatorname{dim} M>5$, which is not $C R$ equivalent to the standard sphere $\mathbf{S}^{2 n+1}$. Let $\theta$ be a strictly pseudoconvex pseudohermitian structure in $\{J\}$ which has positive Webster scalar curvature. Define

$$
\operatorname{can}(J)=\mathcal{A}_{\theta}^{2} \theta
$$

where

$$
\begin{aligned}
& \mathcal{A}_{\theta}(x)=\lim _{y \rightarrow x}\left|G_{\theta}(x, y)-\rho_{\theta}(x, y)\right|^{\frac{1}{Q-2}}, \\
& \rho_{\theta}(x, y)=\frac{1}{\phi(x) \phi(y)} \cdot \frac{C_{Q}}{\left\|x y^{-1}\right\|^{Q-2}},
\end{aligned}
$$

if $\theta=\phi^{\frac{4}{Q-2}} \theta_{\mathbf{H}}$ on a neighborhood $U$ of $x$. Here $G_{\theta}$ is the Green function of the conformal SubLaplacian $L_{\theta}, C_{Q}$ is a constant defined by (3.7), and $\theta_{\mathbf{H}}$ is the standard contact form on $\mathbf{H}^{n}$. Then, $\operatorname{can}(J)$ is a well-defined $C^{\infty}$ contact form and depends only on the CR structure J. 
Here $\rho_{\theta}(x, y)$ is the singular part of the Green function $G_{\theta}(x, y)$. We call can $(J)$ the canonical contact form of Habermann-Jost type since it is the CR analogue of canonical metric on locally conformally flat manifolds constructed by Habermann and Jost. Similar CR invariant contact form can be defined on open and bounded sets in $\mathbf{H}^{n}$. See [L] for the Euclidean case.

We require that $\operatorname{dim} M>5$ because we use the CR positive mass theorem in the proof of the theorem, which is available only in such dimensions now [Li1].

Let $\Gamma$ be a discrete subgroup of $\operatorname{Aut}_{C R}\left(\mathbf{S}^{2 n+1}\right)=P U(n+1,1)$. A point $\xi \in$ $\mathbf{S}^{2 n+1}$ is called a limit point of $\Gamma$ if there exist $\xi^{\prime} \in \mathbf{B}^{2 n+2}=\left\{z \in \mathbf{C}^{n+1} \|\left. z\right|^{2}<1\right\}$ and $\left\{\gamma_{k}\right\}, \gamma_{k} \in \Gamma$, such that $\xi=\lim _{k} \longrightarrow \infty \gamma_{k} \xi^{\prime}$. The limit set $\Lambda(\Gamma)$ of all limit points is closed and invariant under $\Gamma$.

$$
\Omega(\Gamma)=\mathbf{S}^{2 n+1} \backslash \Lambda(\Gamma)
$$

is the maximal domain in $\mathbf{S}^{2 n+1}$ on which $\Gamma$ acts properly discontinuously, which is called the discontinuity domain of $\Gamma$. $\Gamma$ is called a Kleinian group if $\Omega(\Gamma)$ is non empty. A Kleinian group $\Gamma$ is called elementary if $\Lambda(\Gamma)$ contains at most two points. A discrete subgroup $\Gamma$ is called convex cocompact if the quotient of convex hull of $\Lambda(\Gamma)$ is compact.

Let $J_{\mathbf{S}}$ be the standard complex structure on $\mathbf{S}^{2 n+1}$ induced from $\mathbf{C}^{n+1}$ and $\theta_{\mathbf{S}}$ be the standard contact form on $\mathbf{S}^{2 n+1}$. For $\gamma \in P U(n+1,1)$, we denote by $\left|\gamma^{\prime}\right|$ the positive function on $\mathbf{S}^{2 n+1}$ such that

$$
\gamma^{*} \theta_{\mathbf{S}}=\left|\gamma^{\prime}\right|^{2} \theta_{\mathbf{s}}
$$

For a convex cocompact group $\Gamma$, we can calculate the canonical contact form of Habermann-Jost type on $\Omega(\Gamma) / \Gamma$ as follows.

Theorem 1.2. Let $\Gamma$ be a convex cocompact subgroup of $P U(n+1,1)$, and

$$
\pi_{\Gamma}: \Omega(\Gamma) \longrightarrow \Omega(\Gamma) / \Gamma
$$

be the canonical projection. Suppose $\Omega(\Gamma) / \Gamma$ is scalar positive, then

$$
\pi_{\Gamma}^{*} \operatorname{can}\left(J_{\Gamma}\right)=\mathcal{A}_{\Gamma}^{2} \theta_{\mathbf{S}},
$$

where $J_{\Gamma}$ is the complex structure on $\Omega(\Gamma) / \Gamma$ induced by the canonical projection $\pi_{\Gamma}$, and

$$
\mathcal{A}_{\Gamma}(x)=\left(\sum_{\gamma \in \Gamma \backslash\{1\}}\left|\gamma^{\prime}(x)\right|^{\frac{Q-2}{2}} G_{\mathbf{S}}(x, \gamma x)\right)^{\frac{1}{Q-2}},
$$

and $G_{\mathbf{S}}$ is the Green function of the conformal SubLaplacian $L_{\theta_{\mathbf{S}}}$ on $\mathbf{S}^{2 n+1}$.

The critical exponent $\delta(\Gamma)$ of a Kleinian group $\Gamma$ is defined to be

$$
\delta(\Gamma)=\inf \left\{s>0 ; \sum_{\gamma \in \Gamma} e^{-\frac{1}{2} s \cdot d(x, \gamma y)}<\infty\right\}
$$

where $x, y$ are in the unit ball $\mathbf{B}^{2 n+2} \subset \mathbf{C}^{n+1}$ and $d(\cdot, \cdot)$ is the complex hyperbolic distance on $\mathbf{B}^{2 n+2} . \delta(\Gamma)$ is independent of the particular choice of points $x, y$. Our definition of critical exponent is different from that in $[\mathrm{C}][\mathrm{EMM}]$ with a factor $\frac{1}{2}$. 
Theorem [C] [EMM]. For any convex cocompact Kleinian group $\Gamma$ of $S U(n+1,1)$, there exists a probability measure $\mu_{\Gamma}$ supported on $\Lambda(\Gamma)$ such that

$$
\gamma^{*} \mu_{\Gamma}=\left|\gamma^{\prime}\right|^{\delta(\Gamma)} \mu_{\Gamma}
$$

for each $\gamma \in \Gamma$. If $\Gamma$ is non-elementary, measure satisfying (1.12) is unique up to a constant multiple.

This measure coincides, up to a constant multiple, with the $\delta(\Gamma)$ dimensional Carnot-Hausdorffmeasure restricted to the limit set $\Lambda(\Gamma)$, i.e. there exist constants $c$ and $r_{0}$ such that if $x \in \Lambda(\Gamma)$ and $r<r_{0}$, then

$$
\frac{1}{c} \leq \frac{\mu_{\Gamma}\left(B_{r}(x)\right)}{r^{\delta(\Gamma)}} \leq c,
$$

where $B_{r}(x)$ is the ball in $\mathbf{S}^{2 n+1}$ under the Carnot distance.

Such measure is called Patterson-Sullivan measure. Define a $C^{\infty}$ function $\Psi_{\Gamma}$ on $\Omega(\Gamma)$ by

$$
\Psi_{\Gamma}(x)=\left(\int_{\Lambda(\Gamma)} G_{\mathbf{S}}^{p}(x, y) d \mu_{\Gamma}(y)\right)^{\frac{1}{p}}, \quad p=\frac{2 \delta(\Gamma)}{Q-2} .
$$

It can be shown that the contact form

$$
\hat{\theta}_{\Gamma}=\Psi_{\Gamma}^{\frac{4}{Q-2}} \theta_{\mathbf{S}}
$$

on $\Omega(\Gamma)$ is invariant under $\Gamma$, and hence induces a spherical contact form on $\Omega(\Gamma) / \Gamma$. This is the CR generalization of Nayatani's canonical metric in conformal geometry. We call $\hat{\theta}_{\Gamma}$ the canonical contact form of Nayatani type. $\hat{\theta}_{\Gamma}$ has remarkable properties and can be also used to prove the following relationship between its Webster scalar curvature and the critical exponent as in [N].

Theorem 1.3. Let $\Gamma$ be a convex cocompact subgroup of $P U(n+1,1)$ such that $\Lambda(\Gamma) \neq\{$ point $\}$. Then, if $\delta(\Gamma)<n$ (respectively, $\delta(\Gamma)=n$, or $\delta(\Gamma)>n$ ), the Webster scalar curvature of $\hat{\theta}_{\Gamma}$ is positive (respectively, zero, or negative) everywhere.

This theorem has the following important corollary.

Corollary 1.4. For a convex cocompact subgroup $\Gamma$ of $P U(n+1,1)$ such that $\Lambda(\Gamma) \neq$ point $\}, \Omega(\Gamma) / \Gamma$ is scalar positive (respectively, scalar vanishing, or scalar negative) if and only if $\delta(\Gamma)<n$ (respectively, $\delta(\Gamma)=n$, or $\delta(\Gamma)>n$ ).

When $\Gamma$ is a convex cocompact subgroup of $S O(n+1,1)$, Nayatani $[\mathrm{N}]$ calculate the curvature term of the Weitzenböck formula for the Hodge Laplacian defined by his canonical metric, and proved the vanishing theorem for the cohomology groups of the group $\Gamma$. This result is generalized by Izeki [I]. It is interesting to find their complex analogue. But their arguments do not seem to work directly in the complex case. 
Similar to the Riemannian case $[\mathrm{H}]$, it is interesting to investigate the structure of the moduli space of scalar positive spherical CR structures. It seems to be possible to develope the theory for convex cocompact subgroups of other rank-1 Lie groups.

The paper is organized as follows. In Sect. 2, we collect basic facts about CR geometry, the Heisenberg group, the complex hyperbolic geometry and discrete subgroups. In Sect. 3, we construct the CR invariant canonical contact form of Habermann-Jost type on scalar positive spherical CR manifolds. In Sect. 4, we construct the canonical contact form of Nayatani type on CR Kleinian manifolds and prove the relationship between the sign of its Webster scalar curvature and the critical exponent. In Sect. 5, we prove that the connected sum of two scalar positive spherical CR manifolds is also scalar positive.

I would like to thank the referees for many valuable suggestions and Dr. Wolfgang Staubach for reading of the manuscript.

\section{Some basic facts}

We collect some basic facts about CR geometry, the Heisenberg group, the complex hyperbolic geometry and discrete subgroups in this section, (cf. [JL1] [EMM, Appendix A]).

Let $M$ be a real $2 n+1$ dimensional orientable $C^{\infty}$ manifold. A $C R$ structure on $M$ is a $n$ dimensional complex subbundle $T_{1,0}$ of the complexified tangent bundle CTM satisfying $T_{1,0} \cap T_{0,1}=\{0\}$, where $T_{0,1}=\overline{T_{1,0}}$, and integrability condition: $\left[Z_{1}, Z_{2}\right] \in C^{\infty}\left(M, T_{1,0}\right)$ whenever $Z_{1}, Z_{2} \in C^{\infty}\left(M, T_{1,0}\right)$. Set

$$
H=\operatorname{Re}\left\{T_{1,0} \oplus T_{0,1}\right\}
$$

It is the $2 n$ dimensional real horizontal subbundle of TM. $H$ carries a complex structure $J: H \longrightarrow H$ satisfying $J^{2}=-\mathrm{id}_{H}$ and $T_{1,0}=\operatorname{ker}\left(J-i \cdot \operatorname{id}_{\mathbf{C} H}\right)$, $T_{0,1}=\operatorname{ker}\left(J+i \cdot \operatorname{id}_{\mathbf{C} H}\right)$.

Let $E \subset T^{*} M$ denote the real line bundle $H^{\perp}$. Because we assume $M$ to be orientable, the complex structure $J$ induces an orientation on $H$. $E$ has a globally non-vanishing section $\theta$.

We can define a Hermitian form on $T_{1,0}$ associated with $\theta$ by

$$
\langle V, \bar{W}\rangle_{\theta}=-2 i d \theta(V \wedge \bar{W})
$$

which is called the Levi form of $\theta$. Note that

$$
\langle V, \bar{W}\rangle_{\theta}=2 d \theta(V \wedge \overline{J W}) .
$$

In this form $\langle\cdot, \cdot\rangle_{\theta}$ extends by complex linearity to a symmetric form on $\mathbf{C H}$, which is real on $H$. If $\langle\cdot, \cdot\rangle_{\theta}$ is positive definite, $(M, \theta)$ is said to be strictly pseudoconvex. The inner product $\langle\cdot, \cdot\rangle_{\theta}$ determines an isomorphism $H^{*} \cong H$, which in turn 
determines a dual form $\langle\cdot, \cdot\rangle_{\theta}^{*}$ on $H^{*} .\langle\cdot, \cdot\rangle_{\theta}^{*}$ can be naturally extended to $T^{*} M$. This defines a norm $|\omega|_{\theta}$ on the space of real $1-$ forms $\omega$ by

$$
|\omega|_{\theta}^{2}=\langle\omega, \omega\rangle_{\theta}^{*}=2 \sum_{j=1}^{n}\left|\omega\left(Z_{j}\right)\right|^{2},
$$

where $Z_{1}, \cdots, Z_{n}$ form an orthonormal basis for $H$ with respect to the Levi form $\langle\cdot, \cdot\rangle_{\theta}$.

For a pseudohermitian structure $\theta$ on a strictly pseudoconvex CR manifold $(M, J)$, there is a unique vector field $T$, which is transversal to $H$, defined by

$$
\theta(T)=1, \quad d \theta(T \wedge \cdot)=0 .
$$

A mapping $f:\left(M_{1}, J_{1}\right) \longrightarrow\left(M_{2}, J_{2}\right)$ is called a Cauchy-Riemann mapping (or CR mapping) if $f_{*} J_{1}=J_{2} f_{*}$. If $f$ is a CR diffeomorphism, then $f_{*} H_{1}=H_{2}$, where $H_{1}$ and $H_{2}$ are real horizontal subbundles of $T M_{1}$ and $T M_{2}$, respectively. Then, $f^{*} \theta_{2}$ is a globally non-vanishing section of $E_{1}=H_{1}^{\perp}$ if $\theta_{2}$ is a globally nonvanishing section of $E_{2}=H_{2}^{\perp}$. For any globally non-vanishing section $\theta_{1}$ of $E_{1}$, we have $f^{*} \theta_{2}=\phi \theta_{1}$ for some non-vanishing function $\phi$ on $M_{1}$. It is easy to see that $\phi>0$ when $\theta_{1}$ and $\theta_{2}$ are both strictly pseudoconvex. So, CR diffeomorphism $f$ is conformal.

In [W], Webster shows that there exists a natural connection on the bundle $T_{1,0}$ adapted to a pseudohermitian structure $\theta$. Let $\theta^{\alpha}$ be an admissible coframe, i.e. $(1,0)$-forms $\theta^{\alpha}$ form a basis for $T_{1,0}^{*}$ such that $\theta^{\alpha}(T)=0$ for all $\alpha=1, \cdots, n$. The integrability condition implies

$$
d \theta=i g_{\alpha \bar{\beta}} \theta^{\alpha} \wedge \theta^{\bar{\beta}}
$$

for some Hermitian matrix of functions $\left(g_{\alpha \bar{\beta}}\right)$, which is positive definite if $(M, \theta)$ is strictly pseudoconvex. In this case, $\theta$ is a contact form, i.e. $\theta \wedge(d \theta)^{n}$ is nowhere vanishing. Webster showed that there are uniquely determined 1 -forms $\omega_{\alpha}{ }^{\beta}$ and $\tau^{\beta}$ on $M$ satisfying

$$
\begin{aligned}
& d \theta^{\beta}=\theta^{\alpha} \wedge \omega_{\alpha}^{\beta}+\tau^{\beta} \\
& \omega_{\alpha \bar{\beta}}+\omega_{\bar{\beta} \alpha}=d g_{\alpha \bar{\beta}} \\
& \tau_{\alpha} \wedge \theta^{\alpha}=0,
\end{aligned}
$$

where we use $\left(g_{\alpha \bar{\beta}}\right)$ to raise and lower indices, e.g. $\omega_{\alpha \bar{\beta}}=\omega_{\alpha}{ }^{\gamma} g_{\gamma \bar{\beta}}$. Let

$$
\Omega_{\beta}^{\alpha}=d \omega_{\beta}^{\alpha}+\omega_{\beta}^{\gamma} \wedge \omega_{\gamma}^{\alpha} \text {. }
$$

Webster showed that $\Omega_{\beta}{ }^{\alpha}$ could be written as

$$
\Omega_{\beta}{ }^{\alpha}=R_{\beta}{ }^{\alpha} \rho \bar{\sigma}^{\rho} \theta^{\rho} \wedge \theta^{\bar{\sigma}}+W_{\beta}{ }^{\alpha}{ }_{\rho} \theta^{\rho} \wedge \theta+W_{\beta \bar{\rho}}^{\alpha} \theta^{\bar{\rho}} \wedge \theta .
$$

The Webster-Ricci tensor of $(M, \theta)$ has components $R_{\alpha \bar{\beta}}=R_{\rho \alpha \bar{\beta}}^{\rho}$. The Webster scalar curvature is

$$
R_{\theta}=g^{\alpha \bar{\beta}} R_{\alpha \bar{\beta}}
$$


The CR Yamabe problem is to find a contact form $\tilde{\theta}=u^{\frac{4}{Q-2}} \theta, u>0$, which is conformal to the given contact form $\theta$, such that $R_{\tilde{\theta}} \equiv$ constant. This problem is solved by Lee and Jerison [JL1]-[JL3] in the case that $(M, \theta)$ not locally spherical and $n>1$, and by Gamara and Yacoub [G] [GY] in the remaining case.

We can define the SubLaplacian $\triangle_{\theta}$ associated with a strictly pseudoconvex contact form $\theta$ by

$$
\int_{M} \triangle_{\theta} u \cdot v \theta \wedge(d \theta)^{n}=\int_{M}\langle d u, d v\rangle_{\theta}^{*} \theta \wedge(d \theta)^{n} .
$$

Since evidently, $|\theta|_{\theta}=0,\langle\cdot, \cdot\rangle_{\theta}^{*}$ is degenerate on $T^{*} M$ and the operator $\triangle_{\theta}$ is subelliptic rather than elliptic.

Proposition 2.1 [Le, Proposition 4.10]. If $u \in C_{0}^{\infty}$, then $\triangle_{\theta} u=-u_{\alpha}{ }^{\alpha}-u_{\bar{\alpha}}{ }^{\bar{\alpha}}$.

By using the regularity result of $\triangle_{\theta}$ in Proposition 5.7 in [JL1] repeatedly, we have

Proposition 2.2. Let $U$ be a relatively compact open set in $M$. Suppose $f, g \in$ $C^{\infty}(U)$, and $\triangle_{\theta} u+g u=f$ in the distribution sense on $U, u \in L^{2}(U)$. Then, $u \in C^{\infty}(U)$.

The following maximum principle follows from the Harnack inequality (Proposition 5.12 in [JL1]) by standard argument [GT, pp. 188].

Proposition 2.3 (The maximum principle). Let $U \subset M$ be open, $g \geq 0$ on $U$, $P=\triangle_{\theta}+g$. Suppose $u \in C^{2}(U)$ such that $P u \geq 0$ (resp., $\left.P u \leq 0\right)$. Then $u$ can't achieve a non-positive minimum (resp., non-negative maximum) in $U$ unless it is a constant.

The simplest CR manifold is the Heisenberg group $\mathbf{H}^{n}$, whose underlying manifold is $\mathbf{C}^{n} \times \mathbf{R}$, with coordinates $(z, t)$. Its multiplication is given by

$$
(z, t) \cdot\left(z^{\prime}, t^{\prime}\right)=\left(z+z^{\prime}, t+t^{\prime}+2 \operatorname{Im} z \bar{z}^{\prime}\right),
$$

where $z \bar{z}^{\prime}=\sum_{j=1}^{n} z_{j} \bar{z}_{j}^{\prime}$. It's obvious that $(z, t)^{-1}=(-z,-t)$. Define a norm on $\mathbf{H}^{n}$ by

$$
\|(z, t)\|=\left(|z|^{4}+t^{2}\right)^{\frac{1}{4}},
$$

and the dilation by

$$
\delta_{\lambda}(z, t)=\left(\lambda z, \lambda^{2} t\right), \quad \text { for } \lambda>0 .
$$

Note that $\left\|\delta_{\lambda}(z, t)\right\|=\lambda\|(z, t)\|$. For $(z, t),\left(z^{\prime}, t^{\prime}\right) \in \mathbf{H}^{n}, d\left((z, t),\left(z^{\prime}, t^{\prime}\right)\right)=\|(z, t)^{-1}$. $\left(z^{\prime}, t^{\prime}\right) \|$ defines a distance on $\mathbf{H}^{n}$. The vector fields

$$
Z_{j}=\frac{\partial}{\partial z_{j}}+i \bar{z}_{j} \frac{\partial}{\partial t},
$$


$j=1, \cdots, n$, are left invariant vector fields on $\mathbf{H}^{n}$. The subbundle $T_{1,0}$ is $\operatorname{span}\left\{Z_{1}\right.$, $\left.\cdots, Z_{n}\right\}$. Let

$$
\theta_{\mathbf{H}}=d t+\sum_{j=1}^{n} i\left(z_{j} d \bar{z}_{j}-\bar{z}_{j} d z_{j}\right)
$$

be the standard contact form on $\mathbf{H}^{n}$, which is left invariant. Since $\delta_{\lambda}^{*} \theta_{\mathbf{H}}=\lambda^{2} \theta_{\mathbf{H}}$,

$$
\delta_{\lambda}^{*}\left(\theta_{\mathbf{H}} \wedge\left(d \theta_{\mathbf{H}}\right)^{n}\right)=\lambda^{2 n+2} \theta_{\mathbf{H}} \wedge\left(d \theta_{\mathbf{H}}\right)^{n},
$$

which means the homogeneous dimension of $\mathbf{H}^{n}$ is $Q=2 n+2$. Let $\left(x_{1}, \cdots\right.$, $\left.x_{2 n}, t\right)$ be coordinates of $\mathbf{H}^{n}$, then

$$
\theta_{\mathbf{H}} \wedge d \theta_{\mathbf{H}}^{n}=n ! 2^{2 n} d x_{1} \wedge \cdots \wedge d x_{2 n} \wedge d t .
$$

The Levi form is given by

$$
\left\langle Z_{j}, \bar{Z}_{k}\right\rangle_{\theta_{\mathbf{H}}}=-2 i d \theta_{\mathbf{H}}\left(Z_{j} \wedge \bar{Z}_{k}\right)=2 \delta_{j k} .
$$

Thus, $\frac{1}{\sqrt{2}} Z_{1}, \cdots, \frac{1}{\sqrt{2}} Z_{n}$ form an orthogonal basis of $T_{1,0}$. Hence, for $u \in C^{1}\left(\mathbf{H}^{n}\right)$,

$$
d u=\frac{\partial u}{\partial t} \theta_{\mathbf{H}}+\sum_{j=1}^{n} Z_{j} u d z_{j}+\bar{Z}_{j} u d \bar{z}_{j},
$$

and

$$
|d u|_{\theta_{\mathbf{H}}}^{2}=\sum_{j=1}^{n}\left|Z_{j} u\right|^{2}
$$

if $u$ is real valued. Then,

$$
\triangle_{\theta_{\mathbf{H}}}=-\frac{1}{2} \sum_{j=1}^{n}\left(Z_{j} \bar{Z}_{j}+\bar{Z}_{j} Z_{j}\right)
$$

The Cayley transformation between the unit ball $B^{2 n+2}=\left\{\left(\zeta, \zeta_{n+1}\right) \in \mathbf{C}^{n} \times\right.$ $\left.\left.\mathbf{C}|| \zeta\right|^{2}<1\right\}$ and the Siegel upper half space $\mathcal{D}=\left\{\left(z, z_{n+1}\right) \in \mathbf{C}^{n} \times \mathbf{C} \mid \operatorname{Im} z_{n+1}>\right.$ $\left.|z|^{2}\right\}$ is given by

$$
z_{n+1}=i \frac{1-\zeta_{n+1}}{1+\zeta_{n+1}}, \quad z=\frac{\zeta}{1+\zeta_{n+1}} .
$$

Hence, we have the stereographic projection $F: \mathbf{S}^{2 n+1} \backslash\{(0, \cdots, 0,-1)\} \longrightarrow \mathbf{H}^{n}$ given by

$$
t=\operatorname{Re} z_{n+1}=\frac{2 \operatorname{Im} \zeta_{n+1}}{\left|1+\zeta_{n+1}\right|^{2}}, \quad z=\frac{\zeta}{1+\zeta_{n+1}} .
$$

Sphere $\mathbf{S}^{2 n+1}$ has the standard contact form

$$
\theta_{\mathbf{S}}=i(\bar{\partial}-\partial)\left(\left|\zeta_{1}\right|^{2}+\cdots\left|\zeta_{n+1}\right|^{2}\right)
$$


with $R_{\theta_{\mathbf{S}}} \equiv \frac{n(n+1)}{2}$. F is a conformal mapping, i.e.

$$
F^{*}\left(\frac{4}{|w+i|^{2}} \theta_{\mathbf{H}}\right)=\theta_{\mathbf{S}}
$$

where $w=t+i|z|^{2}$.

Consider the Hermitian form

$$
Q\left(\zeta, \zeta^{\prime}\right)=\zeta_{1} \bar{\zeta}_{1}^{\prime}+\cdots+\zeta_{n+1} \bar{\zeta}_{n+1}^{\prime}-\zeta_{n+2} \bar{\zeta}_{n+2}^{\prime}
$$

on $\mathbf{C}^{n+2}$ and the following subset in $\mathbf{C}^{n+2}$ :

$$
\begin{aligned}
& V_{0}=\left\{\zeta \in \mathbf{C}^{n+2} \mid Q(\zeta, \zeta)=0\right\}, \\
& V_{-}=\left\{\zeta \in \mathbf{C}^{n+2} \mid Q(\zeta, \zeta)<0\right\}
\end{aligned}
$$

Let $P: \mathbf{C}^{n+2} \backslash\{0\} \longrightarrow \mathbf{C P}^{\mathbf{n}+\mathbf{1}}$ be the canonical projection onto the complex projective space. Then, $\mathbf{H}_{\mathbf{C}}^{\mathbf{n}+\mathbf{1}}=P\left(V_{-}\right)$is the complex hyperbolic space and the group $U(n+1,1)$ is a subgroup of $G L(n+2, \mathbf{C})$ whose elements preserving the Hermitian form $Q$ defined by (2.27). The action of $U(n+1,1)$ on $V_{-}$ induces an action on $\mathbf{H}_{\mathbf{C}}^{\mathbf{n}+\mathbf{1}}$ with kernel isomorphic to $S^{1}$. Set $P U(n+1,1)=$ $U(n+1,1) /$ kernel. $S U(n+1,1)$ is the group of unimodular transformations preserving the Hermitian form $Q$. Its center consists of $n+2$ transformations: $\zeta_{j}^{*}=\varepsilon \zeta_{j}$, $\varepsilon^{n+2}=1, j=1, \cdots, n+2$. Then $S U(n+1,1) /$ center acts on $P\left(V_{0}\right)$ effectively and $P U(n+1,1)=S U(n+1,1) /$ center. Almost all results for discrete subgroups of $S U(n+1,1)$ hold for discrete subgroups of $P U(n+1,1)$.

We can obtain a model for the complex hyperbolic space in the unit ball by setting $z_{j}=\zeta_{j} / \zeta_{n+2}, j=1,2, \cdots, n+1$. Then $\mathbf{H}_{\mathbf{C}}^{\mathbf{n}+\mathbf{1}}=P\left(V_{-}\right)$is just the ball $\mathbf{B}^{2 n+2}=\left\{\left.z \in \mathbf{C}^{n+1}\left|\sum_{j=1}^{n}\right| z_{j}\right|^{2}<1\right\}$. Under this identification, $P U(n+1,1)$ acts on $\mathbf{B}^{2 n+2}$ as

$$
\gamma(z)=\left(\frac{\gamma(z, 1)_{1}}{\gamma(z, 1)_{n+2}}, \cdots, \frac{\gamma(z, 1)_{n+1}}{\gamma(z, 1)_{n+2}}\right),
$$

for $\gamma \in P U(n+1,1)$ and $z \in \mathbf{B}^{2 n+2}$, where we denote by $\gamma(z, 1)_{j}$ the $\mathrm{j}$-th component of $\gamma(z, 1)$. The fundamental invariant is given by

$$
(X, Y)=\frac{1-\langle X, Y\rangle}{\left(1-|X|^{2}\right)^{\frac{1}{2}}\left(1-|Y|^{2}\right)^{\frac{1}{2}}}
$$

with $\langle X, Y\rangle=\sum_{j=1}^{n+1} X_{j} \bar{Y}_{j}$, and

$$
|(X, Y)|=\cosh \left(\frac{1}{2} d(X, Y)\right),
$$

for $X, Y \in \mathbf{B}^{2 n+2}$, where $d(X, Y)$ is the complex hyperbolic distance between $X$ and $Y$. 
Define a family of measures

$$
\mu_{s}=\frac{\sum_{\gamma \in \Gamma} e^{-\frac{1}{2} s \cdot d(0, \gamma y)} \delta_{\gamma y}}{\sum_{\gamma \in \Gamma} e^{-\frac{1}{2} s \cdot d(y, \gamma y)}}
$$

for $s>\delta(\Gamma)$, where $\delta_{\gamma y}$ is the Dirac measure at point $\gamma y$. The definition of the measure $\mu_{s}$ does not depend on $y \in \mathbf{B}^{2 n+2}$. The Patterson-Sullivan measure is the weak limit of these measures,

$$
\mu_{\Gamma}(x)=\lim _{s \longrightarrow \delta(\Gamma)+} \mu_{s}(x) .
$$

The theorem in the introduction about the Patterson-Sullivan measure is proved in $[C]$ and [EMM] except for (1.12). We prove (1.12) now.

Proof of (1.12). Note that our definition of the critical exponent (1.11) is different from that in $[\mathrm{C}][\mathrm{EMM}]$ with a factor $\frac{1}{2}$. By Lemma A5.10 in [EMM],

$$
\gamma^{*} d \mu_{\Gamma}(z)=\frac{1}{\left|\gamma(z, 1)_{n+2}\right|^{\delta(\Gamma)}} d \mu_{\Gamma}(z)
$$

for $z \in \mathbf{S}^{2 n+1}$ and $\gamma \in P U(n+1,1)$. It is sufficient to check that $\left|\gamma^{\prime}(z)\right|=\frac{1}{\left|\gamma(z, 1)_{n+2}\right|}$, i.e.

$$
\gamma^{*} \theta_{\mathbf{S}}(z)=\frac{1}{\left|\gamma(z, 1)_{n+2}\right|^{2}} \theta_{\mathbf{S}}(z)
$$

Note

$$
d \gamma(z)=\left(\cdots, \frac{\gamma(d z, 0)_{j}}{\gamma(z, 1)_{n+2}}-\frac{\gamma(z, 1)_{j} \gamma(d z, 0)_{n+2}}{\gamma(z, 1)_{n+2}^{2}}, \cdots\right),
$$

by the linearity of $\gamma$. We have, for $z \in \mathbf{S}^{2 n+1}$,

$$
\begin{aligned}
\sum_{j=1}^{n+1} \gamma(z)_{j} d \overline{\gamma(z)}_{j} & =\sum_{j=1}^{n+1} \frac{\gamma(z, 1)_{j} \bar{\gamma}(d \bar{z}, 0)_{j}}{\left|\gamma(z, 1)_{n+2}\right|^{2}}-\sum_{j=1}^{n+1} \frac{\left|\gamma(z, 1)_{j}\right|^{2} \bar{\gamma}(d \bar{z}, 0)_{n+2}}{\left|\gamma(z, 1)_{n+2}\right|^{2} \bar{\gamma}(\bar{z}, 1)_{n+2}} \\
& =\sum_{j=1}^{n+1} \frac{\gamma(z, 1)_{j} \bar{\gamma}(d \bar{z}, 0)_{j}}{\left|\gamma(z, 1)_{n+2}\right|^{2}}-\frac{\gamma(z, 1)_{n+2} \bar{\gamma}(d \bar{z}, 0)_{n+2}}{\left|\gamma(z, 1)_{n+2}\right|^{2}} \\
& =\sum_{j=1}^{n+1} \frac{z_{j} d \bar{z}_{j}}{\left|\gamma(z, 1)_{n+2}\right|^{2}},
\end{aligned}
$$

by $\sum_{j=1}^{n+1}\left|\gamma(z, 1)_{j}\right|^{2}-\left|\gamma(z, 1)_{n+2}\right|^{2}=|z|^{2}-1=0$ for $\gamma \in U(n+1,1)$. (2.35) follows from the definition of $\theta_{\mathbf{S}}$ in (2.25) and (2.37). 
Proposition 2.4. Let $\Gamma$ be a convex cocompact subgroup of $P U(n+1,1)$. Then, its critical exponent

$$
\delta(\Gamma)=\inf \left\{s>0 ; \sum_{\gamma \in \Gamma}\left|\gamma^{\prime}(\xi)\right|^{s}<\infty\right\},
$$

for any fixed $\xi \in \Omega(\Gamma)$.

Proof. Without loss of generality, we can assume $\xi=(1,0, \cdots, 0)$. Let $\gamma \in$ $P U(n+1,1)$. It follows from (2.30)-(2.31) that

$$
e^{d(0, \gamma 0)} \sim \frac{1}{1-|\gamma(0)|^{2}}=\frac{1}{1-\sum_{j=1}^{n+1} \frac{\left|\gamma_{j(n+2)}\right|^{2}}{\left|\gamma_{(n+2)(n+2)}\right|^{2}}}=\left|\gamma_{(n+2)(n+2)}\right|^{2},
$$

by

$$
\sum_{j=1}^{n+1}\left|\gamma_{j(n+1)}\right|^{2}-\left|\gamma_{(n+2)(n+2)}\right|^{2}=-1
$$

Since $\xi \in \Omega(\Gamma)$, there exists $\varepsilon>0$ such that the Euclidean distant between $\xi$ and $\gamma(0)$ for any $\gamma \in \Gamma$ is larger than $\varepsilon$. We can assume that $\left|\gamma(0)_{1}\right|=\left|\frac{\gamma_{1(n+2)}}{\gamma_{(n+2)(n+2)}}\right|=$ $\left|\frac{\gamma_{(n+2) 1}}{\gamma_{(n+2)(n+2)}}\right|<1-\varepsilon$. So,

$$
\begin{aligned}
\left|\gamma^{\prime}(\xi)\right|^{2} & =\frac{1}{\left|\gamma(1,0, \cdots, 0,1)_{n+2}\right|^{2}} \\
& =\frac{1}{\left|\gamma_{(n+2) 1}-\gamma_{(n+2)(n+2)}\right|^{2}} \sim \frac{1}{\left|\gamma_{(n+2)(n+2)}\right|^{2}}
\end{aligned}
$$

by (2.35) and the above facts. Therefore, $e^{-d(0, \gamma(0))} \sim\left|\gamma^{\prime}(\xi)\right|^{2}$ for fixed $\xi \in \Omega(\Gamma)$. (2.38) follows.

It is well known that $\operatorname{Aut}_{C R} \mathbf{S}^{2 n+1}=P U(n+1,1)$ and $\operatorname{Aut}_{C R} \mathbf{H}^{n}$ is the stabilizer of $P U(n+1,1)$ fixing the south pole [BS], [KT]. Thus, $P U(n+1,1)$ is generated by the following CR transformations on $\mathbf{H}^{n}$,

(1) dilations:

$$
\delta_{a}(z, t)=\left(a z, a^{2} t\right), \quad a>0 ;
$$

(2) left translations:

$$
\tau_{(z, t)}:\left(z^{\prime}, t^{\prime}\right) \longrightarrow(z, t) \cdot\left(z^{\prime}, t^{\prime}\right), \quad(z, t),\left(z^{\prime}, t^{\prime}\right) \in \mathbf{H}^{n} ;
$$

(3) unitary transformations:

$$
U_{A}:(z, t) \longrightarrow(A z, t), \quad A \in U(n)
$$

(4) inversion:

$$
R:(z, t) \longrightarrow\left(\frac{-z}{|z|^{2}-i t}, \frac{-t}{|z|^{4}+t^{2}}\right)
$$


Each compact spherical $\mathrm{CR}$ manifold $M$ has a $C R$ developing mapping [BS] [KT]:

$$
\Phi: \tilde{M} \longrightarrow \mathbf{S}^{2 n+1}
$$

where $\tilde{M}$ is the universal covering of $M$, and $\Phi$ is unique up to composition with elements in $\operatorname{Aut}_{C R}\left(\mathbf{S}^{2 n+1}\right)=P U(n+1,1)$. The developing mapping $\Phi$ induces a group homomorphism:

$$
\Phi_{*}: \pi_{1}(M) \longrightarrow P U(n+1,1) .
$$

The developing mapping $\Phi$ is not always injective. If it is, $\pi_{1}(M)$ is a discrete subgroup of $P U(n+1,1)$ and $\tilde{M}$ is CR equivalent to an open set $\Omega \subset \mathbf{S}^{2 n+1}$, and hence $M$ is $\Omega / \pi_{1}(M)$.

Since any CR mapping $f: U \longrightarrow V$ between open sets $U, V \subset \mathbf{S}^{2 n+1}$ is the restriction to $U$ of a mapping holomorphic in a domain containing $U$, the following theorem follows from Theorem 1.1 in [BS].

Theorem 2.5 (Liouville-type Theorem). If $f$ is a $C R$ diffeomorphism from an open set $U \subset \mathbf{H}^{n}$ to another open set $V \subset \mathbf{H}^{n}$, then $f$ is the restriction to $U$ of an element in $P U(n+1,1)$.

To define the Carnot-Hausdorff measure, we should replace the Euclidean balls in the classical definition of Hausdorff measure by nonisotropic balls. Let $B(\xi, r)$ denote the nonisotropic ball $\left\{y \in \mathbf{H}^{n} ;\left\|y^{-1} \cdot \xi\right\| \leq r\right\}$. Define

$$
m_{\delta, \varepsilon}(E)=\inf \left\{\sum_{v} r_{v}^{\delta} \mid E \subset \cup_{\nu} B\left(\xi_{v}, r_{v}\right), r_{v} \leq \varepsilon\right\},
$$

where infimum is taken over all coverings $B\left(\xi_{v}, r_{v}\right)$ of $E$. The $\delta$ dimensional Carnot-Hausdorff measure of a Borel set $E \subset \mathbf{H}^{n}$ is given by

$$
m_{\delta}(E)=\lim _{\varepsilon \longrightarrow 0} m_{\delta, \varepsilon}(E)
$$

The Carnot-Hausdorff dimension of a Borel set $E \subset \mathbf{H}^{n}$ is the number

$$
d=\inf \left\{\delta \mid m_{\delta}(E)=0\right\} .
$$

We will need the following $C R$ positive mass theorem in the spherical CR setting [Li1].

Theorem 2.6. Let $(M, \theta)$ be a compact, pseudohermitian, spherical CR manifold with positive Webster scalar curvature, where $\operatorname{dim} M=2 n+1$. Then,

1. For each $p \in M$, there exists a local CR diffeomorphism $C_{p}$ from a neighborhood of $p$ to the Heisenberg group $\mathbf{H}^{n}$ such that $C_{p}(p)=\infty,\left(C_{p}^{-1}\right)^{*}\left(G_{p}^{t-2} \theta\right)=$ $h^{t-2} \theta_{\mathbf{H}}$, where $t=2+\frac{2}{n} ; h=1+A_{p} \rho^{-2 n}+o\left(\rho^{-2 n-1}\right) ; G_{p}$ is the Green function for the conformal SubLaplacian $L_{\theta}$ with pole at $p ; \rho$ is the norm on $\mathbf{H}^{n}$; and these CR local diffeomorphisms are parameterized by the $C R$ automorphism group of $\mathbf{S}^{2 n+1}$ fixing $\infty$. We call $A_{p}$ CR mass.

2. Sign $\left(A_{p}\right)$ is independent of the choice of coordinates (or CR developing maps) and so it is a CR invariant. When $n \geq 3$, it is nonnegative and is zero if and only if $(M, \theta)$ is $C R$ equivalent to the standard complex unit sphere $\mathbf{S}^{2 n+1}$. 


\section{Canonical contact form of Habermann-Jost type}

Proposition 3.1. Let $M$ be a connected compact manifold of dimension $2 n+1$, $J$ be a strictly pseudoconvex CR structure on $M$. Then, there is one and only one of the following cases holding: $\{J\}$ contains a contact form with either (1) positive, or (2) negative, or (3) vanishing Webster scalar curvature.

Proof. The proof is similar to the Riemannian case [H, p. 17]. Choose a $\theta \in\{J\}$. The conformal SubLaplacian $L_{\theta}$ is selfadjoint and subelliptic. Its spectra are bounded from below. Let $\lambda_{1}$ be the first eigenvalue of $L_{\theta}$ and $\phi$ be a corresponding eigenfunction. Then, $\phi$ is $C^{\infty}$ and nowhere vanishing, which will be proved later. We assume $\phi>0$. Then, $\theta^{\prime}=\phi^{\frac{4}{Q-2}} \theta$ is also a contact form in $\{J\}$ whose Webster scalar curvature $R_{\theta^{\prime}}=\lambda_{1} \phi^{-\frac{4}{Q-2}}$ by the transformation formula (1.2). Hence, $R_{\theta^{\prime}}>0$ (or $R_{\theta^{\prime}}<0$, or $R_{\theta^{\prime}} \equiv 0$ ) if $\lambda_{1}>0$ (or $\lambda_{1}<0$, or $\left.\lambda_{1}=0\right)$.

On the other hand, if a contact form $\hat{\theta} \in\{J\}$ has Webster scalar curvature $R_{\hat{\theta}}>0$ (or $<0$, or $\equiv 0$ ), then the first eigenvalue $\hat{\lambda}_{1}$ of $L_{\hat{\theta}}$ obviously satisfies $\hat{\lambda}_{1}>0$ (or $<0$, or $=0$ ).

At last, by the transformation formula (1.1), the sign of the first eigenvalue of $L_{\theta}$ does not depend on the choice of $\theta \in\{J\}$. The result follows.

It remains to prove that the eigenfunction $\phi$ is $C^{\infty}$ and nowhere vanishing. It can be proved exactly the same way as Theorem 4.4 in [A] in the Riemannian case. We sketch it here. Define $\left|\nabla_{\theta} u\right|=\left(\sum_{j=1}^{n}\left|Z_{j} u\right|^{2}\right)^{\frac{1}{2}}$, where $\left\{Z_{1}, \cdots, Z_{n}\right\}$ is an orthonormal basis of $T_{1,0}$ under strictly pseudoconvex pseudohermitian structure $\theta$. Since any orthonormal basis of $T_{1,0}$ can be transformed to $\left\{Z_{1}, \cdots, Z_{n}\right\}$ by an unitary transformation, $\left|\nabla_{\theta} u\right|$ does not depend on the choice of such basis. Define the nonisotropic Sobolev space $W^{2,1}(M)=\left\{u ; u,|\nabla u| \in L^{2}(M)\right\}$. It can be shown that $|u| \in W^{2,1}(M)$ if $u \in W^{2,1}(M)$ by calculus of weak differentiation [GT, Sect. 7.4]. Then, the first eigenvalue $\lambda_{1}=\inf _{u \in \mathcal{W}_{+}}\left\{\left\|\nabla_{\theta} u\right\|_{L^{2}}^{2}+\int_{M} R_{\theta}|u|^{2}\right\}$, where $\mathcal{W}_{+}=\left\{u \in W^{2,1}(M) ;\|u\|_{L^{2}}=1, u \geq 0\right\}$. Let $\left\{u_{j}\right\}$ be a minimizing sequence in $\mathcal{W}_{+}$. There exist $\phi \in \mathcal{W}_{+}$and a subsequence of $\left\{u_{j}\right\}$ converging to $\phi$ in $L^{2}(M)$ and weakly in $W^{2,1}(M) . \lambda_{1}$ is attained by $\phi$ and the Euler-Lagrange equation yields $L_{\theta} \phi=\lambda_{1} \phi$. Then, $\phi$ is $C^{\infty}$ and positive by regularity result in Proposition 2.2 and the maximum principle in Proposition 2.3, respectively. The proposition is now proved.

Remark. (1) This proposition does not exclude the possibility that the scalar positive CR manifolds have contact forms with Webster scalar curvature vanishing or negative somewhere.

(2) Our definition of function $\mathcal{A}_{\theta}$ in (1.5) is a little bit different from that in [H], since if we choose $\theta=\theta_{\mathbf{H}}$ locally for $\theta \in\{J\}$ as in $[\mathrm{H}], \theta$ doesn't have positive Webster scalar curvature. The existence of Green function can not directly follow from the invertibility of $L_{\theta}$. 
A continuous function $G_{\theta}: M \times M \backslash \operatorname{diag} M \longrightarrow \mathbf{R}$ is called a Green function of the conformal SubLaplacian $L_{\theta}$ if

$$
\int_{M} G_{\theta}(x, y) L_{\theta} u(y) \theta \wedge(d \theta)^{n}(y)=u(x)
$$

for all $u \in C_{0}^{\infty}(M)$.

Proposition 3.2. Let $(M, J)$ be strictly pseudoconvex $C R$ manifold, $\theta \in\{J\}$ and $G_{\theta}$ be a Green function of the conformal SubLaplacian $L_{\theta}$. Then $G_{\tilde{\theta}}$ given by

$$
G_{\tilde{\theta}}=\frac{1}{\phi(x) \phi(y)} G_{\theta}(x, y)
$$

is a Green function of the conformal SubLaplacian $L_{\tilde{\theta}}$ with $\tilde{\theta}=\phi^{\frac{4}{Q-2}} \theta$.

Proof. Since

$$
d \tilde{\theta}=\frac{4}{Q-2} \phi^{\frac{6-Q}{Q-2}} d \phi \wedge \theta+\phi^{\frac{4}{Q-2}} d \theta
$$

we have

$$
\tilde{\theta} \wedge(d \tilde{\theta})^{n}=\phi^{\frac{2 Q}{Q-2}} \theta \wedge(d \theta)^{n}
$$

Therefore,

$$
\begin{aligned}
\int_{M} \frac{1}{\phi(x) \phi(y)} G_{\theta}(x, y)\left(L_{\tilde{\theta}} u\right)(y) \tilde{\theta} \wedge(d \tilde{\theta})^{n}(y) \\
=\frac{1}{\phi(x)} \int_{M} G_{\theta}(x, y) L_{\theta}(\phi u)(y) \theta \wedge(d \theta)^{n}(y)=u(x),
\end{aligned}
$$

for any $u \in C_{0}^{\infty}(M)$, by the transformation formula (1.1). The proposition is proved.

From now on in this section, we assume $(M, \theta)$ to be compact and scalar positive. So, $G_{\theta}$ always exists by using Beals-Greiner calculus [BG] since $L_{\theta}$ is invertible in $L^{2}(M)$ by $R_{\theta}>0$. The Green function of the conformal SubLaplacian $L_{\theta}$ is unique in this case.

The Green function of the conformal SubLaplacian $L_{\theta_{\mathbf{H}}}$ on the Heisenberg group $\mathbf{H}^{n}$ is [JL1, pp. 180]

$$
G_{\theta_{\mathbf{H}}}(x, y)=\frac{C_{Q}}{\left\|x \cdot y^{-1}\right\|^{Q-2}},
$$

for $x \neq y, x, y \in \mathbf{H}^{n}$, where $\|\cdot\|$ is the norm on $\mathbf{H}^{n}$ defined by (2.13) and constant

$$
C_{Q}=\frac{2^{2-2 n} \pi^{n+1}}{\Gamma\left(\frac{n}{2}\right)^{2}} .
$$

The limit (1.5) exists and is $C^{\infty}$ by the following proposition. In the proof of the following proposition, we also give a direct construction of the Green function of the conformal SubLaplacian $L_{\theta}$ on a spherical CR manifold $(M, J)$ for $\theta \in\{J\}$. 
Proposition 3.3. Let $(M, J)$ be a compact spherical $C R$ manifold, $\theta \in\{J\}$ have positive scalar curvature, and $U$ be a sufficiently small open set. Then the function $G_{\theta}(x, y)-\rho_{\theta}(x, y)$ can be extended to a $C^{\infty}$ function on $U \times U$.

Proof. Suppose $\bar{U} \Subset \tilde{U} \subset \mathbf{H}^{n}$ and $\theta=\phi^{\frac{4}{Q-2}} \theta_{\mathbf{H}}$ on $\tilde{U}$. We choose $\rho$ so small that $B(\eta, \rho) \subset \tilde{U}$ for any $\eta \in U$. We can construct the Green function as follows. For $\xi, \eta \in U$, define

$$
\begin{aligned}
& G_{0}(\xi, \eta)=\tilde{G}_{0}\left(\xi \eta^{-1}\right), \\
& \tilde{G}_{0}(y)=\frac{C_{Q}}{\|y\|^{Q-2}} f(y), \quad y \in \mathbf{H}^{n},
\end{aligned}
$$

where $f \in C_{0}^{\infty}\left(\mathbf{H}^{n}\right)$ satisfying $f \equiv 1$ on $B\left(0, \frac{\rho}{2}\right)$ and $f \equiv 0$ on $B(0, \rho)^{c}$. Denote $Z_{j}=X_{j}-i X_{j+n}, j=1, \cdots, n$. Then, $\triangle_{\theta_{\mathbf{H}}}=-\sum_{j=1}^{2 n} X_{j}^{2}$. Note $L_{\theta_{\mathbf{H}}}=b_{n} \triangle_{\theta_{\mathbf{H}}}$. Then,

$$
\begin{aligned}
L_{\theta_{\mathbf{H}}} \tilde{G}_{0}(y) & =\delta_{0}-b_{n} \sum_{j=1}^{2 n} X_{j}\left(\frac{C_{Q}}{\|y\|^{Q-2}}\right) X_{j} f(y)+\frac{C_{Q}}{\|y\|^{Q-2}} L_{\theta_{\mathbf{H}}} f(y) \\
& =\delta_{0}+\tilde{G}_{1}(y),
\end{aligned}
$$

by $\frac{C_{Q}}{\|y\|^{2-Q}}$ being the fundamental solution of $L_{\theta_{\mathbf{H}}}$ and $X_{j} f \equiv 0$ on $B\left(\xi, \frac{\rho}{2}\right)$, where $\delta_{0}$ is the Dirac function at the origin under the measure $\theta_{\mathbf{H}} \wedge\left(d \theta_{\mathbf{H}}\right)^{n}$. Set $G_{1}(\xi, \cdot)=$ $\tilde{G}_{1}\left(\xi \eta^{-1}\right)$ for $\xi, \eta \in U$. Then, $G_{1}(\xi, \eta) \in C^{\infty}(U \times U)$ and for each $\xi \in U, G_{1}(\xi, \cdot)$ can be natually extended to a smooth function on $M$ satisfying $G_{1}(\xi, \eta)=0$ for $\eta \notin \tilde{U}$. By (1.1),

$$
\begin{aligned}
L_{\theta}\left(\phi(\xi)^{-1} \phi(\cdot)^{-1} G_{0}(\xi, \cdot)\right) & =\phi(\xi)^{-1} \phi(\cdot)^{-\frac{Q+2}{Q-2}} L_{\theta_{\mathbf{H}}} G_{0}(\xi, \cdot) \\
& =\delta_{\xi}+\phi(\xi)^{-1} \phi(\cdot)^{-\frac{Q+2}{Q-2}} G_{1}(\xi, \cdot),
\end{aligned}
$$

on $U$ for $\xi \in U$, where $\delta \xi$ is the Dirac function at point $\xi$ under the measure $\theta \wedge(d \theta)^{n}=\phi^{\frac{2 Q}{Q-2}} \theta_{\mathbf{H}} \wedge\left(d \theta_{\mathbf{H}}\right)^{n}$. Now set

$$
G(\xi, \eta)=\phi(\xi)^{-1} \phi(\eta)^{-1} G_{0}(\xi, \eta)+G_{2}(\xi, \eta)
$$

for $\xi \in U, \eta \in M$, where $G_{2}$ satisfies $L_{\theta} G_{2}(\xi, \cdot)=-\phi(\xi)^{-1} \phi(\cdot)^{-\frac{Q+2}{Q-2}} G_{1}(\xi, \cdot)$. $G_{2}(\xi, \cdot)$ exists since $L_{\theta}$ is invertible in $L^{2}(M) . G_{2}(\xi, \cdot) \in C^{\infty}(U)$ for fixed $\xi$ by regularity of $L_{\theta}$ in Proposition 2.2. $G_{2}(\cdot, \eta)$ is also in $C^{\infty}(U)$ by differentiating $L_{\theta} G_{2}(\xi, \cdot)=G_{1}(\xi, \cdot)$ with respect to variable $\xi$ repeatedly. Then, $L_{\theta} G(\xi, \cdot)=\delta \xi$, i.e., $G(\xi, \eta)$ is the Green function $G_{\theta}(\xi, \eta)$ of $L_{\theta}$. By $(3.11), G_{\theta}(\xi, \eta)-\rho_{\theta}(\xi, \eta) \in$ $C^{\infty}(U \times U)$. It follows from (3.11) that $G(\xi, \eta) \longrightarrow+\infty$ as $\eta \longrightarrow \xi$. By the maximum principle (Proposition 2.3), $G_{\theta}(\xi, \eta)>0$ for all $\xi \neq \eta$.

Proof of Theorem 1.1. Let's verify that $\mathcal{A}_{\theta}$ is independent of the choice of local coordinates and $\mathcal{A}_{\theta}^{2} \theta$ is independent of the choice of $\theta \in\{J\}$. Suppose $\theta, \tilde{\theta} \in\{J\}$ 
and $\tilde{\theta}=\tilde{\phi}^{\frac{4}{Q-2}} \theta$. Let $U \subset M$ be an open set, $\Phi: U \longrightarrow V \subset \mathbf{H}^{n}$ and $\tilde{\Phi}: U \longrightarrow$ $\tilde{V} \subset \mathbf{H}^{n}$ be two coordinates such that

$$
\begin{aligned}
& \theta=\Phi^{*}\left(\phi_{1}^{\frac{4}{Q-2}} \theta_{\mathbf{H}}\right), \\
& \tilde{\theta}=\tilde{\Phi}^{*}\left(\phi_{2}^{\frac{4}{Q-2}} \theta_{\mathbf{H}}\right),
\end{aligned}
$$

for two positive functions $\phi_{1}, \phi_{2}$. Then, $f=\tilde{\Phi} \circ \Phi^{-1}: V \longrightarrow \tilde{V}$ is a $\mathrm{CR}$ diffeomorphism and

$$
f^{*} \theta_{\mathbf{H}}(\xi)=\phi^{\frac{4}{Q-2}}(\xi) \theta_{\mathbf{H}}(\xi)
$$

for $\xi \in V$, where $\phi(\xi)=\phi_{1}(\xi) \phi_{2}(f(\xi))^{-1} \tilde{\phi}\left(\Phi^{-1}(\xi)\right)$. We claim that, for $\xi, \eta \in V$,

$$
\left\|f(\xi) f(\eta)^{-1}\right\|=\phi^{\frac{1}{Q-2}}(\xi) \phi^{\frac{1}{Q-2}}(\eta)\left\|\xi \eta^{-1}\right\| .
$$

By applying (3.14) to $\xi=\Phi(x), \eta=\Phi(y)$ and $f=\tilde{\Phi} \circ \Phi^{-1}$, we find

$$
\begin{aligned}
\mathcal{A}_{\tilde{\theta}}(x) & =\lim _{y \longrightarrow x}\left|G_{\tilde{\theta}}(x, y)-\frac{C_{Q}\left\|\tilde{\Phi}(x) \tilde{\Phi}(y)^{-1}\right\|^{2-Q}}{\phi_{2}(\tilde{\Phi}(x)) \phi_{2}(\tilde{\Phi}(y))}\right|^{\frac{1}{Q-2}} \\
& =\lim _{y \longrightarrow x}\left|\frac{G_{\theta}(x, y)}{\tilde{\phi}(x) \tilde{\phi}(y)}-\frac{C_{Q}\left\|\Phi(x) \Phi(y)^{-1}\right\|^{2-Q}}{\tilde{\phi}(x) \tilde{\phi}(y) \phi_{1}(\Phi(x)) \phi_{1}(\Phi(y))}\right|^{\frac{1}{Q-2}} \\
& =\tilde{\phi}^{\frac{2}{Q-2}}(x) \lim _{y \longrightarrow x}\left|G_{\theta}(x, y)-\frac{C_{Q}\left\|\Phi(x) \Phi(y)^{-1}\right\|^{2-Q}}{\phi_{1}(\Phi(x)) \phi_{1}(\Phi(y))}\right|^{\frac{1}{Q-2}} \\
& =\tilde{\phi}^{\frac{2}{Q-2}}(x) \mathcal{A}_{\theta}(x) .
\end{aligned}
$$

So,

$$
\mathcal{A}_{\tilde{\theta}}^{2} \tilde{\theta}=\mathcal{A}_{\theta}^{2} \theta
$$

We will use positive mass theorem to show that $\mathcal{A}_{\theta}$ is non-vanishing. Now let $\theta=\phi^{\frac{4}{Q-2}} \theta_{\mathbf{H}}$ locally. Denote by $A(x)$ the smooth function $\lim _{y \rightarrow x}\left(G_{\theta}(x, y)\right.$ $\left.-\rho_{\theta}(x, y)\right)$. Note that

$$
\begin{aligned}
& G_{\theta}(x, y)^{\frac{4}{Q-2}} \theta(y)=\left(\rho_{\theta}(x, y)+A(x)+O\left(\left\|x^{-1} y\right\|\right)\right)^{\frac{4}{Q-2}} \theta(y) \\
& \quad=\frac{\alpha(x)}{\left\|x^{-1} y\right\|^{4}} \cdot\left(1+\beta(x)\left\|x^{-1} y\right\|^{Q-2}+O\left(\left\|x^{-1} y\right\|^{Q-1}\right)\right)^{\frac{4}{Q-2}} \theta_{\mathbf{H}}(y),
\end{aligned}
$$

where $\alpha(x)=\left(C_{Q} \phi(x)^{-1}\right)^{\frac{4}{Q-2}}$ and $\beta(x)=\frac{A(x) \phi(x)^{2}}{C_{Q}}$. Note that the inversion $R$ defined by (2.45) is just $F \circ I \circ F^{-1}$, where $F$ is the stereographic projection defined by (2.24), and $I$ is the CR automorphism of $\mathbf{S}^{2 n+1}$ given by $\left(\xi_{1}, \cdots, \xi_{n+1}\right) \longrightarrow$ 
$\left(\xi_{1}, \cdots, \xi_{n},-\xi_{n+1}\right)$. It follows from the transformation formula (2.26) and the fact $I$ preserving $\theta_{\mathbf{S}}$ that

$$
\left(R^{*} \theta_{\mathbf{H}}\right)(z, t)=\frac{1}{\|(z, t)\|^{4}} \theta_{\mathbf{H}}(z, t) .
$$

(cf. [JL1, p. 192]). Now Let $C_{x}=R \circ \delta_{r} \circ \tau_{x^{-1}}$, a CR mapping on a neighborhood of $x$ to $\mathbf{H}^{n}$, where $\delta_{r}$ is the dilation, $r=\alpha(x)^{-\frac{1}{2}}$. It is easy to see that $C_{x}(x)=\infty$ and

$$
\begin{aligned}
& \left(\left(C_{x}^{-1}\right)^{*} G_{\theta}^{\frac{4}{Q-2}} \theta\right)(\tilde{y})=\left(\left(R^{*} \circ \delta_{r^{-1}}^{*} \circ \tau_{x}^{*}\right) G_{\theta}^{\frac{4}{Q-2}} \theta\right)^{\frac{4}{Q-2}}(\tilde{y}) \\
& =\left(1+\beta(x) r^{-Q+2}\|\tilde{y}\|^{-Q+2}+O\left(\|\tilde{y}\|^{-Q+1}\right)\right)^{\frac{4}{Q-2}} \theta \mathbf{H}(\tilde{y}),
\end{aligned}
$$

where $\tilde{y}=C_{x}(y)$. Note $Q=2 n+2$. The CR positive mass theorem (Theorem 2.6) promises $\beta(x) r^{-Q+2}$ to be positive. Therefore, $A(x)$ is positive and $\mathcal{A}_{\theta}$ is nonvanishing.

It remains to check (3.14). By Liouville-type theorem, $f$ is a restriction on $U$ of a CR automorphism of $\mathbf{H}^{n}$. We denote it also by $f$. By the transformation law (1.1), for $\theta=f^{*} \theta_{\mathbf{H}}(\xi)=\phi^{\frac{4}{Q-2}}(\xi) \theta_{\mathbf{H}}(\xi)$,

$$
\begin{aligned}
& L_{\theta_{\mathbf{H}}}\left(\tilde{\phi}^{-1} \tilde{u}\right)(f(\eta))=L_{\theta}\left(\phi^{-1} u\right)(\eta)=\phi^{-\frac{Q+2}{Q-2}}(\eta)\left(L_{\theta_{\mathbf{H}}} u\right)(\eta), \\
& f^{*}\left(\theta_{\mathbf{H}} \wedge\left(d \theta_{\mathbf{H}}\right)^{n}\right)(\eta)=\phi^{\frac{2 Q}{Q-2}}(\eta) \cdot \theta_{\mathbf{H}} \wedge\left(d \theta_{\mathbf{H}}\right)^{n}(\eta) .
\end{aligned}
$$

where $\tilde{\phi}=\phi \circ f^{-1}, \tilde{u}=u \circ f^{-1}$. Therefore, by substituting (3.20) and taking transformation $f(\eta) \longrightarrow \eta^{\prime}$, we have

$$
\begin{aligned}
\int_{\mathbf{H}^{n}} C_{Q} \phi(\xi) \phi(\eta)\left\|f(\xi) f(\eta)^{-1}\right\|^{2-Q} L_{\theta_{\mathbf{H}}} u(\eta) \cdot \theta_{\mathbf{H}} \wedge\left(d \theta_{\mathbf{H}}\right)^{n}(\eta) \\
=\int_{\mathbf{H}^{n}} C_{Q} \phi(\xi)\left\|f(\xi) f(\eta)^{-1}\right\|^{2-Q}\left(L_{\theta_{\mathbf{H}}}\left(\tilde{\phi}^{-1} \tilde{u}\right)\right)(f(\eta)) \\
\quad \cdot f^{*}\left(\theta_{\mathbf{H}} \wedge\left(d \theta_{\mathbf{H}}\right)^{n}\right)(\eta) \\
=\int_{\mathbf{H}^{n}} C_{Q} \phi(\xi)\left\|f(\xi) \eta^{\prime-1}\right\|^{2-Q}\left(L_{\theta_{\mathbf{H}}}\left(\tilde{\phi}^{-1} \tilde{u}\right)\right)\left(\eta^{\prime}\right) \cdot \theta_{\mathbf{H}} \wedge\left(d \theta_{\mathbf{H}}\right)^{n}\left(\eta^{\prime}\right) \\
=u(\xi) .
\end{aligned}
$$

Now, by the uniqueness of positive Green's function of $L_{\theta_{\mathbf{H}}}$, we find that $G_{\theta_{\mathbf{H}}}(\xi, \eta)$ $=C_{Q} \phi(\xi) \phi(\eta)\left\|f(\xi) f(\eta)^{-1}\right\|^{2-Q}$. Thus, (3.14) follows. The theorem is proved.

The following is the invariance of the canonical contact form under CR diffeomorphisms.

Proposition 3.4. Let $\left(M_{1}, J_{1}\right)$ and $\left(M_{2}, J_{2}\right)$ be two connected, compact, scalar positive, spherical CR manifolds with $\operatorname{dim} M_{1}=\operatorname{dim} M_{2}>5$, which are both not $C R$ equivalent to the standard sphere $\mathbf{S}^{2 n+1}$. Suppose $f:\left(M_{1}, J_{1}\right) \longrightarrow\left(M_{2}, J_{2}\right)$ be a locally diffeomorphic CR mapping. Then,

$$
\left|f^{*} \operatorname{can}\left(J_{2}\right)(V)\right| \geq\left|\operatorname{can}\left(J_{1}\right)\left(f_{*} V\right)\right|
$$

for any tangent vector $V \in T M$. 
Proof. Since $f$ is a local diffeomorphism and $M_{1}$ and $M_{2}$ are connected and compact, $f$ is a finite regular covering. Fix $x \in M_{1}$ and choose $\theta_{2} \in\left\{J_{2}\right\}$ such that $\theta_{2}$ has positive Webster scalar curvature. Let $U_{2}$ be a neighborhood of $f(x)$, $\Phi_{2}: U_{2} \longrightarrow V \subset \mathbf{H}^{n}$ be a coordinate transformation and $\theta_{2}=\Phi_{2}^{*}\left(\phi^{\frac{4}{Q-2}} \theta_{\mathbf{H}}\right)$ on $U$ for some positive function $\phi$. Then, $\theta_{1}=f^{*} \theta_{2} \in\left\{J_{1}\right\}$ has positive Webster scalar curvature, $U_{1}=f^{-1}\left(U_{2}\right)$ is a neighborhood of $x, \Phi_{1}=\Phi_{2} \circ f$ is a coordinate transformation and $\theta_{1}=\Phi_{1}^{*}\left(\phi^{\frac{4}{Q-2}} \theta_{\mathbf{H}}\right)$.

By the definition of Green function,

$$
G_{\theta_{2}}(f(x), f(y))=\sum_{\gamma \in \Gamma} G_{\theta_{1}}(x, \gamma y)
$$

for $y \in M_{1}$ and $f(x) \neq f(y)$, where $\Gamma$ is the covering group. It follows that

$$
\begin{aligned}
\mathcal{A}_{\theta_{2}}^{Q-2} & (f(x))=\lim _{y^{\prime} \longrightarrow f(x)}\left|G_{\theta_{2}}\left(f(x), y^{\prime}\right)-\frac{C_{Q}\left\|\Phi_{2}(f(x)) \Phi_{2}\left(y^{\prime}\right)^{-1}\right\|^{2-Q}}{\phi\left(\Phi_{2}(f(x))\right) \phi\left(\Phi_{2}\left(y^{\prime}\right)\right)}\right| \\
& =\lim _{y \longrightarrow x}\left|\sum_{\gamma \in \Gamma} G_{\theta_{1}}(x, \gamma y)-\frac{C_{Q}\left\|\Phi_{1}(x) \Phi_{1}(y)^{-1}\right\|^{2-Q}}{\phi\left(\Phi_{1}(x)\right) \phi\left(\Phi_{1}(y)\right)}\right| \\
& =\lim _{y \rightarrow x}\left|G_{\theta_{1}}(x, y)-\frac{C_{Q}\left\|\Phi_{1}(x) \Phi_{1}(y)^{-1}\right\|^{2-Q}}{\phi\left(\Phi_{1}(x)\right) \phi\left(\Phi_{1}(y)\right)}\right|+\sum_{\gamma \in \Gamma \backslash\{1\}} G_{\theta_{1}}(x, \gamma y) \\
& =\mathcal{A}_{\theta_{1}}^{Q-2}(x)+\sum_{\gamma \in \Gamma \backslash\{1\}} G_{\theta_{1}}(x, \gamma y) \geq \mathcal{A}_{\theta_{1}}^{Q-2}(x) .
\end{aligned}
$$

In the third equality, we use the fact that $G_{\theta_{1}}(x, y)-\frac{C_{Q}\left\|\Phi_{1}(x) \Phi_{1}(y)^{-1}\right\|^{2-Q}}{\phi\left(\Phi_{1}(x)\right) \phi\left(\Phi_{1}(y)\right)}$ and $G_{\theta_{1}}(x, \gamma y)$ are all positive. Hence,

$$
\begin{aligned}
\left|f^{*} \operatorname{can}\left(J_{2}\right)(x)(V)\right| & =\left|\mathcal{A}_{\theta_{2}}^{2}(f(x)) \theta_{2}\left(f_{*} V\right)\right| \\
& \geq\left|\mathcal{A}_{\theta_{1}}^{2}(x) \theta_{1}(V)\right|=\left|\operatorname{can}\left(J_{1}\right)(x)(V)\right|
\end{aligned}
$$

for $V \in T M$.

Since CR mapping preserves orientation, we have the following invariance of $\operatorname{can}(J)$.

Corollary 3.5. Let $\left(M_{1}, J_{1}\right)$ and $\left(M_{2}, J_{2}\right)$ be as in Proposition 3.4. If $f$ is a $C R$ diffeomorphism, then

$$
f^{*} \operatorname{can}\left(J_{2}\right)=\operatorname{can}\left(J_{1}\right)
$$

can $(J)$ is invariant under the CR diffeomorphisms of $(M, J)$.

Let us calculate the canonical contact form of Habermann-Jost type on Kleinian manifolds. Suppose $\theta$ is a spherical contact form on $M=\Omega(\Gamma) / \Gamma$. Note that by the canonical projection $\pi_{\Gamma}: \Omega(\Gamma) \longrightarrow \Omega(\Gamma) / \Gamma, \pi_{\Gamma}^{*} \theta$ is conformally equivalent 
to the restriction of the standard contact form $\theta_{\mathbf{S}}$ of $\mathbf{S}^{2 n+1}$ to $\Omega(\Gamma)$. Let $\hat{\phi}$ be the positive function on $\Omega(\Gamma)$ such that

$$
\pi_{\Gamma}^{*} \theta=\hat{\phi}^{\frac{4}{Q-2}} \theta_{\mathbf{S}}
$$

The Green function of the conformal SubLaplacian $L_{\theta}$ on $\Omega(\Gamma) / \Gamma$ can be expressed by $\hat{\phi}$ and the Green function $G_{\mathbf{S}}$ of the conformal SubLaplacian $L_{\theta_{\mathbf{S}}}$ on $\mathbf{S}^{2 n+1}$ as follows.

Proposition 3.6. Let $M, \hat{\phi}$ and $\pi_{\Gamma}$ be defined as above. Then,

$$
G_{\theta}\left(\pi_{\Gamma}(\xi), \pi_{\Gamma}\left(\xi^{\prime}\right)\right)=\frac{1}{\hat{\phi}(\xi) \hat{\phi}\left(\xi^{\prime}\right)} \sum_{\gamma \in \Gamma}\left|\gamma^{\prime}\left(\xi^{\prime}\right)\right|^{\frac{Q-2}{2}} G_{\mathbf{S}}\left(\xi, \gamma \xi^{\prime}\right),
$$

for $\xi, \xi^{\prime} \in \Omega(\Gamma)$ with $\pi_{\Gamma}(\xi) \neq \pi_{\Gamma}\left(\xi^{\prime}\right)$.

Proof. By (3.2),

$$
\hat{G}\left(\xi, \xi^{\prime}\right)=\frac{1}{\hat{\phi}(\xi) \hat{\phi}\left(\xi^{\prime}\right)} G_{\mathbf{S}}\left(\xi, \xi^{\prime}\right)
$$

is the Green function of the conformal SubLaplacian $L_{\pi_{\Gamma}^{*} \theta}$ on $\left(\Omega(\Gamma), \pi_{\Gamma}^{*} \theta\right)$. Since $\pi_{\Gamma}^{*} \theta$ is invariant under $\Gamma$, we have

$$
\hat{\phi}(\gamma \xi)\left|\gamma^{\prime}(\xi)\right|^{\frac{Q-2}{2}}=\hat{\phi}(\xi), \quad \text { for } \quad \xi \in \Omega(\Gamma)
$$

by the definition (1.7) of $\left|\gamma^{\prime}\right|$. Thus, the right side of (3.28) is

$$
\sum_{\gamma \in \Gamma} \hat{G}\left(\xi, \gamma \xi^{\prime}\right)
$$

which is equal to the left side of (3.28). It remains to prove that

$$
\sum_{\gamma \in \Gamma}\left|\gamma^{\prime}\left(\xi^{\prime}\right)\right|^{\frac{Q-2}{2}} G_{\mathbf{S}}\left(\xi, \gamma \xi^{\prime}\right)<\infty
$$

for all $\xi, \xi^{\prime} \in \Omega(\Gamma)$ with $\pi_{\Gamma}(\xi) \neq \pi_{\Gamma}\left(\xi^{\prime}\right)$. Since $M$ is scalar positive, we have $\delta(\Gamma)<n=\frac{Q-2}{2}$ by Theorem 1.3 (We can use Theorem 1.3 here since its proof is independent of Theorem 1.1 and Theorem 1.2), which implies that

$$
\sum_{\gamma \in \Gamma}\left|\gamma^{\prime}\left(\xi^{\prime}\right)\right|^{\frac{Q-2}{2}}<\infty
$$

by Proposition 2.4. On the other hand, since $\Gamma$ acts properly discontinuously on $\Omega(\Gamma)$, there exists a constant $c\left(\xi, \xi^{\prime}\right)$ such that $d\left(\xi, \gamma \xi^{\prime}\right) \geq c\left(\xi, \xi^{\prime}\right)$ for all $\gamma \in \Gamma$. Thus,

$$
G_{\mathbf{S}}\left(\xi, \gamma \xi^{\prime}\right)<C c\left(\xi, \xi^{\prime}\right)^{2-Q}<\infty
$$

for some constant $C>0$. (3.32) is proved. This completes the proof of the proposition. 
Proof of Theorem 1.2. Let $\theta$ be a contact form on $\Omega(\Gamma) / \Gamma$ in $\left\{J_{\Gamma}\right\}$ and $\hat{\phi}$ is a $C^{\infty}$ function on $\Omega(\Gamma)$ such that (3.27) holds on $\Omega(\Gamma)$. Let $\xi \in \Omega(\Gamma)$. Let $F_{-\xi}$ : $\mathbf{S}^{2 n+1} \backslash\{-\xi\} \longrightarrow \mathbf{H}^{n}$ be the stereographic projection, which is the composition of an unitary transformation mapping $-\xi$ to $(0, \cdots, 0,-1)$ and the standard stereographic projection (2.24), and let the function $\phi_{S}$ be defined by

$$
\theta_{\mathbf{S}}=F_{-\xi}^{*}\left(\phi_{S}^{\frac{4}{Q-2}} \theta_{\mathbf{H}}\right)
$$

Then, $F_{-\xi} \circ \pi_{\Gamma}^{-1}$ locally defines a coordinate transformation near $\pi_{\Gamma}(\xi)$ and

$$
\theta=\left(F_{-\xi} \circ \pi_{\Gamma}^{-1}\right)^{*}\left(\left(\hat{\phi} \circ F_{-\xi}^{-1} \cdot \phi_{S}\right)^{\frac{4}{Q-2}} \theta_{\mathbf{H}}\right) .
$$

Thus, $\operatorname{can}\left(J_{\Gamma}\right)=\mathcal{A}^{2} \theta$ with $\mathcal{A}\left(\pi_{\Gamma}(\xi)\right)$ equal to

$$
\lim _{\xi^{\prime} \longrightarrow \xi}\left|G_{\theta}\left(\pi_{\Gamma}(\xi), \pi_{\Gamma}\left(\xi^{\prime}\right)\right)-\frac{C_{Q}}{\left(\hat{\phi} \cdot \hat{\phi}_{S}\right)(\xi)\left(\hat{\phi} \cdot \hat{\phi}_{S}\right)\left(\xi^{\prime}\right)}\left\|F_{-\xi}\left(\xi^{\prime}\right)\right\|^{2-Q}\right|^{\frac{1}{Q-2}},
$$

by the definition of $\mathcal{A}$ and $F_{-\xi}(\xi)=(0, \cdots, 0)$. Here $\hat{\phi}_{S}=\phi_{S} \circ F_{-\xi}$. On the other hand,

$$
\begin{gathered}
G_{\theta}\left(\pi_{\Gamma}(\xi), \pi_{\Gamma}\left(\xi^{\prime}\right)\right)-\frac{C_{Q}}{\left(\hat{\phi} \cdot \hat{\phi}_{S}\right)(\xi)\left(\hat{\phi}^{\prime} \cdot \hat{\phi}_{S}\right)\left(\xi^{\prime}\right)}\left\|F_{-\xi}\left(\xi^{\prime}\right)\right\|^{2-Q} \\
=\frac{1}{\hat{\phi}(\xi) \hat{\phi}\left(\xi^{\prime}\right)} \sum_{\gamma \in \Gamma \backslash\{1\}}\left|\gamma^{\prime}\left(\xi^{\prime}\right)\right|^{\frac{Q-2}{2}} G_{\mathbf{S}}\left(\xi, \gamma \xi^{\prime}\right)
\end{gathered}
$$

by Proposition 3.6 and

$$
G_{\mathbf{S}}\left(\xi, \xi^{\prime}\right)=\frac{C_{Q}}{\hat{\phi}_{S}(\xi) \hat{\phi}_{S}\left(\xi^{\prime}\right)}\left\|F_{-\xi}\left(\xi^{\prime}\right)\right\|^{2-Q}
$$

which follows from the transformation formula of Green functions (3.2) and the Green function of $L_{\theta_{\mathbf{H}}}$. Hence,

$$
\mathcal{A}\left(\pi_{\Gamma}(\xi)\right)=\hat{\phi}(\xi)^{-\frac{2}{Q-2}}\left(\sum_{\gamma \in \Gamma \backslash\{1\}}\left|\gamma^{\prime}(\xi)\right|^{\frac{Q-2}{2}} G_{\mathbf{S}}(\xi, \gamma \xi)\right)^{\frac{1}{Q-2}}
$$

Let $\pi_{\Gamma}^{*} \operatorname{can}\left(J_{\Gamma}\right)=\mathcal{A}_{\Gamma}^{2} \theta_{\mathbf{S}}$. Then,

$$
\mathcal{A}_{\Gamma}(\xi)=\hat{\phi}^{\frac{2}{Q-2}} \mathcal{A}\left(\pi_{\Gamma}(\xi)\right)
$$

by (3.27) and $\operatorname{can}\left(J_{\Gamma}\right)=\mathcal{A}^{2} \theta$. Formula (1.10) follows from (3.40) and (3.41). Theorem 1.2 is proved.

Remark. If $\Gamma$ is nontrivial, then $\mathcal{A}_{\Gamma}>0$ everywhere by (1.10). Hence $\operatorname{can}(J)$ is a spherical contact form on $\Omega(\Gamma) / \Gamma$. Namely, Theorem 1.1 holds for scalar positive Kleinian manifolds in any dimensions. 


\section{Canonical contact form of Nayatani type}

In this section, we construct another canonical contact form $\hat{\theta}_{\Gamma}$, which is the CR generalization of Nayatani's canonical metric in conformal geometry, and calculate its curvature. Since

$$
G_{\mathbf{S}}\left(\gamma \xi, \gamma \xi^{\prime}\right)=\left|\gamma^{\prime}(\xi)\right|^{-\frac{Q-2}{2}}\left|\gamma^{\prime}\left(\xi^{\prime}\right)\right|^{-\frac{Q-2}{2}} G_{\mathbf{S}}\left(\xi, \xi^{\prime}\right)
$$

by the definition of $\left|\gamma^{\prime}\right|$ in (1.7) and the transformation formula of Green functions (3.2), we have

$$
\begin{aligned}
\Psi_{\Gamma}(\gamma \xi) & =\left(\int_{\Lambda(\Gamma)} G_{\mathbf{S}}^{\frac{2 \delta(\Gamma)}{Q-2}}\left(\gamma \xi, \xi^{\prime}\right) d \mu_{\Gamma}\left(\xi^{\prime}\right)\right)^{\frac{Q-2}{2 \delta(\Gamma)}} \\
& =\left(\int_{\Lambda(\Gamma)} G_{\mathbf{S}}^{\frac{2 \delta(\Gamma)}{Q-2}}\left(\gamma \xi, \gamma \xi^{\prime}\right) d \gamma^{*} \mu_{\Gamma}\left(\xi^{\prime}\right)\right)^{\frac{Q-2}{2 \delta(\Gamma)}} \\
& =\left(\int_{\Lambda(\Gamma)}\left|\gamma^{\prime}(\xi)\right|^{-\delta(\Gamma)} G_{\mathbf{S}}^{\frac{2 \delta(\Gamma)}{Q-2}}\left(\xi, \xi^{\prime}\right) d \mu_{\Gamma}\left(\xi^{\prime}\right)\right)^{\frac{Q-2}{2 \delta(\Gamma)}} \\
& =\left|\gamma^{\prime}(\xi)\right|^{-\frac{Q-2}{2}} \Psi_{\Gamma}(\xi),
\end{aligned}
$$

by using the transformation formula (1.12) for Patterson-Sullivan measure. Therefore, $\hat{\theta}_{\Gamma}$ defined by (1.15) is invariant under the action of $\Gamma$. It is obvious nonzero and induces a spherical contact form on $\Omega(\Gamma) / \Gamma$.

Example. Let $\Lambda(\Gamma)$ consists one point. $\delta(\Gamma)=0$ in this case. When $\Lambda(\Gamma)$ is a single point $y$, the Patterson-Sullivan measure $\mu_{\Gamma}$ is the Dirac measure at $y$, and therefore $\hat{\theta}_{\Gamma}=G_{\mathbf{S}}^{\frac{4}{Q-2}}(\cdot, y) \theta_{\mathbf{S}}$. It is easy to verify that $\hat{\theta}_{\Gamma}$ is the standard contact form $\theta_{\mathbf{H}}$.

Proof of Theorem 1.3. We will calculate the curvature of $\hat{\theta}_{\Gamma}$, which is defined by (1.14) and (1.15), locally on $\mathbf{H}^{n}$. Without loss of generality, we can assume the south pole $(0, \cdots, 0,-1)$ of sphere $\mathbf{S}^{2 n+1}$ contained in $\Omega(\Gamma)$. Let $\xi \in \Omega(\Gamma)$. Under the stereographic projection $F$ defined by (2.24),

$$
G_{\mathbf{S}}\left(F^{-1}(\xi), F^{-1}\left(\xi^{\prime}\right)\right)=\left(\frac{4}{|w+i|^{2}}\right)^{-\frac{Q-2}{4}}\left(\frac{4}{\left|w^{\prime}+i\right|^{2}}\right)^{-\frac{Q-2}{4}} G_{\theta_{\mathbf{H}}}\left(\xi, \xi^{\prime}\right)
$$

by (2.26) and (3.2), where

$$
\begin{aligned}
& \xi=(z, t), \quad \xi^{\prime}=\left(z^{\prime}, t^{\prime}\right) \in \mathbf{H}^{n}, \\
& w=t+i|z|^{2}, \quad w^{\prime}=t^{\prime}+i\left|z^{\prime}\right|^{2} .
\end{aligned}
$$

Define the set $\tilde{\Lambda}(\Gamma)$ and the measure $\tilde{\mu}_{\Gamma}$ on $\mathbf{H}^{n}$ by

$$
\begin{aligned}
& \tilde{\Lambda}(\Gamma)=F(\Lambda(\Gamma)), \\
& \tilde{\mu}_{\Gamma}(\xi)=\left(\frac{4}{|w+i|^{2}}\right)^{-\frac{\delta(\Gamma)}{2}} \mu_{\Gamma}\left(F^{-1}(\xi)\right),
\end{aligned}
$$


and the contact form $\tilde{\theta}_{\Gamma}$ on $\mathbf{H}^{n}$ by

$$
\tilde{\theta}_{\Gamma}=\left(\pi_{\Gamma} \circ F^{-1}\right)^{*} \hat{\theta}_{\Gamma} .
$$

By the stereographic projection $F, \Gamma$ induces an action on $\mathbf{H}^{n}$. It is easy to see that the set $\tilde{\Lambda}(\Gamma)$ and the contact form $\tilde{\theta}_{\Gamma}$ on $\mathbf{H}^{n}$ are both invariant under the action of $\Gamma$. Note that $\pi_{\Gamma} \circ F^{-1}$ is locally a CR diffeomorphism. So, the Webster scalar curvature of $\tilde{\theta}_{\Gamma}(\xi)$ is the same as that of $\hat{\theta}_{\Gamma}$ at the point $\pi_{\Gamma} \circ F^{-1}(\xi)$.

Let us calculate the Webster scalar curvature of $\tilde{\theta}_{\Gamma}(\xi)$ now. If we write

$$
\tilde{\theta}_{\Gamma}(\xi)=u^{\frac{4}{Q-2}}(\xi) \theta_{\mathbf{H}}(\xi),
$$

then,

$$
\begin{aligned}
u(\xi) & =\left(\frac{4}{|w+i|^{2}}\right)^{\frac{Q-2}{4}}\left(\int_{\Lambda(\Gamma)} G_{\mathbf{S}}^{p}\left(F^{-1}(\xi), \zeta\right) d \mu_{\Gamma}(\zeta)\right)^{\frac{1}{p}} \\
& =\left(\int_{\tilde{\Lambda}(\Gamma)} G_{\theta_{\mathbf{H}}}^{p}\left(\xi, \xi^{\prime}\right) d \tilde{\mu}_{\Gamma}\left(\xi^{\prime}\right)\right)^{\frac{1}{p}}
\end{aligned}
$$

by (4.3)-(4.5), (2.26) and the definition of $\hat{\theta}_{\Gamma}$, where $p=\frac{2 \delta(\Gamma)}{Q-2}$. Now denote

$$
\begin{aligned}
& \tilde{\theta}_{\Gamma}(x)=e^{2 f(x)} \theta_{\mathbf{H}}(x), \\
& \phi(x, y)=G_{\theta_{\mathbf{H}}}(x, y)^{\frac{2}{2-Q}}=C_{Q}^{\frac{2}{2-Q}}\left\|x y^{-1}\right\|^{2},
\end{aligned}
$$

for $x, y \in \mathbf{H}^{n}$. Then,

$$
f(x)=\frac{1}{\delta(\Gamma)} \log \left(\int_{\tilde{\Lambda}(\Gamma)} \phi(x, y)^{-\delta(\Gamma)} d \tilde{\mu}_{\Gamma}(y)\right),
$$

and the transformation formula of the Webster scalar curvature (Proposition 5.15 in [JL1]) is

$$
R_{\tilde{\theta}_{\Gamma}}=e^{-2 f}\left(Q \triangle_{\theta_{\mathbf{H}}} f-\frac{Q}{2}(Q-2) \sum_{j=1}^{n}\left|Z_{j} f\right|^{2}\right) .
$$

Note

$$
Z_{j} f(x)=-\int_{\tilde{\Lambda}(\Gamma)} \phi_{y}^{-1}(x) Z_{j} \phi_{y}(x) d \nu(y),
$$

where $\phi_{y}(\cdot)=\phi(\cdot, y)$ and let measure

$$
d \nu(y)=\left(\int_{\tilde{\Lambda}(\Gamma)} \phi_{y}^{-\delta} d \tilde{\mu}_{\Gamma}(y)\right)^{-1} \phi_{y}^{-\delta} d \tilde{\mu}_{\Gamma}(y) .
$$


Here $\delta=\delta(\Gamma)$. Direct manipulation gives

$$
\begin{aligned}
\bar{Z}_{j} Z_{j} f= & -\int_{\tilde{\Lambda}(\Gamma)} \phi_{y}^{-1} \bar{Z}_{j} Z_{j} \phi_{y} d \nu+(\delta+1) \int_{\tilde{\Lambda}(\Gamma)} \phi_{y}^{-2} \bar{Z}_{j} \phi_{y} Z_{j} \phi_{y} d \nu \\
& -\delta \int_{\tilde{\Lambda}(\Gamma)} \phi_{y}^{-1} Z_{j} \phi_{y} d \nu \int_{\tilde{\Lambda}(\Gamma)} \phi_{y}^{-1} \bar{Z}_{j} \phi_{y} d \nu .
\end{aligned}
$$

Note $\triangle_{\theta_{\mathbf{H}}}=-\frac{1}{2} \sum_{j=1}^{n}\left(\bar{Z}_{j} Z_{j}+Z_{j} \bar{Z}_{j}\right)$. We find that

$$
\begin{aligned}
& \triangle_{\theta_{\mathbf{H}}} f-\frac{Q-2}{2} \sum_{j=1}^{n}\left|Z_{j} f\right|^{2}=-\int_{\tilde{\Lambda}(\Gamma)} \phi_{y}^{-1} \triangle_{\theta_{\mathbf{H}}} \phi_{y} d \nu \\
& \quad-(\delta+1) \int_{\tilde{\Lambda}(\Gamma)} \phi_{y}^{-2} \sum_{j=1}^{n}\left|Z_{j} \phi_{y}\right|^{2} d \nu+\delta \sum_{j=1}^{n}\left|\int_{\tilde{\Lambda}(\Gamma)} \phi_{y}^{-1} Z_{j} \phi_{y} d \nu\right|^{2} \\
& \quad-\frac{Q-2}{2} \sum_{j=1}^{n}\left|\int_{\tilde{\Lambda}(\Gamma)} \phi_{y}^{-1} Z_{j} \phi_{y} d \nu\right|^{2} \\
& =\left(\frac{Q}{2}-1-\delta\right)\left(\int_{\tilde{\Lambda}(\Gamma)} \phi_{y}^{-2} \sum_{j=1}^{n}\left|Z_{j} \phi_{y}\right|^{2} d \nu-\sum_{j=1}^{n}\left|\int_{\tilde{\Lambda}(\Gamma)} \phi_{y}^{-1} Z_{j} \phi_{y} d \nu\right|^{2}\right) \\
& \quad-\frac{Q}{2} \int_{\tilde{\Lambda}(\Gamma)} \phi_{y}^{-2} \sum_{j=1}^{n}\left|Z_{j} \phi_{y}\right|^{2} d \nu-\int_{\tilde{\Lambda}(\Gamma)} \phi_{y}^{-1} \triangle_{\theta_{\mathbf{H}} \phi_{y} d \nu .}
\end{aligned}
$$

Note that

$$
\triangle_{\theta_{\mathbf{H}}} \phi_{y}=\frac{2}{2-Q} G_{\theta_{\mathbf{H}}}^{\frac{Q}{2-Q}} \triangle_{\theta_{\mathbf{H}}} G_{\theta_{\mathbf{H}}}-\frac{2 Q}{(2-Q)^{2}} G_{\theta_{\mathbf{H}}}^{\frac{2 Q-2}{2-Q}} \sum_{j=1}^{n}\left|Z_{j} G_{\theta_{\mathbf{H}}}\right|^{2}
$$

by the definition of $\phi_{y}$ in (4.9), and

$$
\sum_{j=1}^{n}\left|Z_{j} \phi_{y}\right|^{2}=\frac{4}{(2-Q)^{2}} G_{\theta_{\mathbf{H}}}^{\frac{2 Q}{2-Q}} \sum_{j=1}^{n}\left|Z_{j} G_{\theta_{\mathbf{H}}}\right|^{2}
$$

Moreover, $\triangle_{\theta_{\mathbf{H}}} G_{\theta_{\mathbf{H}}}(x, y)=0$ for $x \neq y$. The last two terms in (4.15) are cancelled. Thus,

$$
\begin{aligned}
R_{\tilde{\theta}_{\Gamma}}= & Q(n-\delta) e^{-2 f}\left(\int_{\tilde{\Lambda}(\Gamma)} \phi_{y}^{-2} \sum_{j=1}^{n}\left|Z_{j} \phi_{y}\right|^{2} d \nu\right. \\
& \left.-\sum_{j=1}^{n}\left|\int_{\tilde{\Lambda}(\Gamma)} \phi_{y}^{-1} Z_{j} \phi_{y} d \nu\right|^{2}\right) .
\end{aligned}
$$

Let be $A=\left(A_{j k}\right)$ the Hermitian matrix with

(4.19) $A_{j k}=\int_{\tilde{\Lambda}(\Gamma)} \phi_{y}^{-2} Z_{j} \phi_{y} \bar{Z}_{k} \phi_{y} d \nu-\int_{\tilde{\Lambda}(\Gamma)} \phi_{y}^{-1} Z_{j} \phi_{y} d \nu \int_{\tilde{\Lambda}(\Gamma)} \phi_{y}^{-1} \bar{Z}_{k} \phi_{y} d \nu$. 
Since $\int_{\tilde{\Lambda}(\Gamma)} d \nu=1$, it is easy to see that $A$ is non-negative by the Cauchy-Schwarz's inequality, i.e. $\operatorname{Tr} A(x) \geq 0$. Then,

$$
R_{\tilde{\theta}_{\Gamma}}=(2 n+2)(n-\delta) e^{-2 f} \operatorname{Tr} A(x) .
$$

Hence, Theorem 1.3 follows from fact that $\operatorname{Tr} A(x)$ is nowhere vanishing on $\Omega(\Gamma)$ by the following lemma.

Lemma 4.1. If

$$
\operatorname{Tr} A(x)=\int_{\tilde{\Lambda}(\Gamma)} \phi_{y}^{-2} \sum_{j=1}^{n}\left|Z_{j} \phi_{y}\right|^{2} d \nu-\sum_{j=1}^{n}\left|\int_{\tilde{\Lambda}(\Gamma)} \phi_{y}^{-1} Z_{j} \phi_{y} d \nu\right|^{2}
$$

vanishes at some point $x \in \tilde{\Omega}(\Gamma)$ and $\delta(\Gamma) \neq n$, then the limit set $\tilde{\Lambda}(\Gamma)$ is exactly one point, and A vanishes identically.

Proof. We still work on $\mathbf{H}^{n}$ as in the proof of Theorem 1.3. Note that the support of Patterson-Sullivan measure $\tilde{\mu}_{\Gamma}$ coincides with the support of $\delta(\Gamma)$-dimensional Carnot-Hausdorff measure $m_{\delta(\Gamma)}$ on $\tilde{\Lambda}(\Gamma)$. Suppose $\operatorname{Tr} A\left(x_{0}\right)=0$ for some $x_{0} \in$ $\tilde{\Omega}(\Gamma)=\mathbf{H}^{n} \backslash \tilde{\Lambda}(\Gamma)$. This is equivalent to the vector $v\left(x_{0}, y\right)=\left(\phi_{y}^{-1} Z_{1} \phi_{y}, \cdots\right.$, $\phi_{y}^{-1} Z_{n} \phi_{y}$ ) being a constant vector at $x_{0}$ for $\tilde{\mu}_{\Gamma}$ almost all $y$ in $\tilde{\Lambda}(\Gamma)$. For $x_{0}=$ $\left(z^{\prime}, t^{\prime}\right) \in \tilde{\Omega}(\Gamma)$ and $y=(z, t) \in \tilde{\Lambda}(\Gamma), \phi_{y}=C_{Q}^{\frac{2}{2-Q}}\|(\hat{z}, \hat{t})\|^{2}$, where $(\hat{z}, \hat{t})=$ $\left(z^{\prime}, t^{\prime}\right)^{-1}(z, t)$, and the $j$-th component of $v\left(x_{0}, y\right)$ is

$$
v_{j}=\phi_{y}^{-1} Z_{j} \phi_{y}=\frac{\left(|\hat{z}|^{2}+i \hat{t}\right) \overline{\hat{z}}_{j}}{|\hat{z}|^{4}+\hat{t}^{2}}
$$

by $Z_{j}$ left invariant for $j=1, \cdots, n$.

Now suppose $\delta(\Gamma)>0$. We can find a Lebegues point $y_{0}$ of the limit set $\tilde{\Lambda}(\Gamma)$. Namely, there exists constants $c$ and $r_{0}>0$ such that the Carnot-Hausdorff measure

$$
m_{\delta(\Gamma)}\left(B\left(y_{0}, r\right) \cap \tilde{\Lambda}(\Gamma)\right) \geq c r^{\delta(\Gamma)} .
$$

for all $r<r_{0}$, where $B\left(y_{0}, r\right)=\left\{\xi \in \mathbf{H}^{n} ;\left\|y_{0} \xi^{-1}\right\|<r\right\}$. Note $R_{\tilde{\theta}_{\Gamma}}\left(x_{0}\right)=0$ by (4.20). Since $\tilde{\theta}_{\Gamma}$ is invariant under the action of $\Gamma, R_{\tilde{\theta}_{\Gamma}}$ is invariant under the action of $\Gamma$. So we find that $R_{\tilde{\theta}_{\Gamma}}\left(\gamma x_{0}\right)=0$ for all $\gamma \in \Gamma$. Therefore, by (4.20) again, $\operatorname{Tr} A\left(\gamma x_{0}\right)=0$ for all $\gamma \in \Gamma$. Note the fundamental domain of $\Gamma$ is compact. We can find a sequence $\gamma_{n} \in \Gamma$ such that $P_{n}=\gamma_{n} x_{0} \longrightarrow y_{0}$. From above, for each $n$, $v\left(P_{n}, y\right)$ is a constant vector for $\tilde{\mu}_{\Gamma}$ almost all $y$ in $\tilde{\Lambda}(\Gamma)$. Thus, there exists a subset $\hat{\Lambda} \subset \tilde{\Lambda}(\Gamma)$ such that $\tilde{\mu}_{\Gamma}(\hat{\Lambda})=\tilde{\mu}_{\Gamma}(\tilde{\Lambda}(\Gamma))$ and

$$
v\left(P_{n}, y\right)=v\left(P_{n}, y^{\prime}\right)
$$

for any $y, y^{\prime} \in \hat{\Lambda}$ and any $n$. We can not promise $y_{0} \in \hat{\Lambda}$. However, since $y_{0}$ is a Lebegues point, we can choose a sequence $y_{m} \in \hat{\Lambda}$ such that $y_{m}$ are all different and different from $y_{0}$, and $y_{m} \longrightarrow y_{0}$ as $m \longrightarrow \infty$. So,

$$
v\left(P_{n}, y_{m}\right)=v\left(P_{n}, y_{1}\right), \quad m, n=1,2, \cdots .
$$


But

$$
\|v(x, y)\|^{2}=\sum_{j=1}^{n}\left|v_{j}\right|^{2}=\frac{|\hat{z}|^{2}}{|\hat{z}|^{4}+\hat{t}^{2}} \leq\left(|\hat{z}|^{4}+\hat{t}^{2}\right)^{-\frac{1}{2}} \longrightarrow+\infty
$$

as $\left\|x^{-1} y\right\| \longrightarrow 0$, where $(\hat{z}, \hat{t})=x^{-1} y$. If we let $m$ and $n$ tend to infinity, i.e. $P_{n} \longrightarrow y_{0}$ and $y_{m} \longrightarrow y_{0}$ in (4.25), the left side of (4.25) tends to infinity while the right side of (4.25) remains finite. This is a contradiction. If $\delta(\Gamma)=0$ and $\Lambda(\Gamma)$ is not a single point, we can find similar contradiction. The lemma is now proved.

Remark. If we assume $x_{0}$ to be the origin. Then $\left\|v\left(x_{0},(z, t)\right)\right\|=\sum_{j=1}^{n}\left|v_{j}\right|^{2}=$ $\left(|z|^{4}+t^{2}\right)^{-1}|z|^{2}$ is a constant for $\tilde{\mu}_{\Gamma}$ almost everywhere $(z, t)$. Thus, if $|z| \neq 0$, $\left(\cdots, \bar{z}_{j}+i \bar{z}_{j} t|z|^{-2}, \cdots\right)$ is a constant vector. Note $\tilde{\Lambda}(\Gamma)$ is a bounded set in $\mathbf{H}^{n}$. If necessarily by translation and rotation, we can assume $(1,0, \cdots, 0) \in \tilde{\Lambda}(\Gamma)$ and the axis $\{(z, t), z=0\} \cap \tilde{\Lambda}(\Gamma)=\emptyset$. Thus, $v\left(x_{0},(z, t)\right)=(1,0, \cdots, 0)$ for all $(z, t) \in \tilde{\Lambda}(\Gamma)$. It follows that almost all points of $\tilde{\Lambda}(\Gamma)$ are contained in the set defined by

$$
\bar{z}_{1}+i \frac{\bar{z}_{1}}{|z|^{2}} t=1, \quad \bar{z}_{j}+i \frac{\bar{z}_{j}}{|z|^{2}} t=0,
$$

$j=2, \cdots, n$. From the later equations in (4.26), $z_{j}=0$ since $1+i t|z|^{-2} \neq 0$. This is a curve. In the case of locally conformally flat manifolds, such argument shows $\Lambda(\Gamma)$ to be a single point. In our case, we need the fact $\operatorname{Tr} A\left(\gamma x_{0}\right)=0$ for all $\gamma \in \Gamma$ to prove $\tilde{\Lambda}(\Gamma)$ being a single point.

\section{Connected sum of of spherical CR manifolds}

Let $M$ be a connected, closed, spherical CR manifold of dimension $2 n+1$ with two punctures $\xi_{1}, \xi_{2}$, or disjoint union of two connected spherical CR manifolds $M_{(1)}, M_{(2)}$ with one puncture $\xi_{i} \in M_{(i)}, i=1,2$, each. Let $J$ be a scalar positive spherical CR structure on $M, U_{1}$ and $U_{2}$ be two disjoint neighborhoods of $\xi_{1}$ and $\xi_{2}$, respectively, and local coordinates

$$
\psi_{i}: U_{i} \longrightarrow B(0,2),
$$

such that $\psi_{i}\left(\xi_{i}\right)=0, i=1,2$, and

$$
B(P, \varepsilon)=\left\{\xi \in \mathbf{H}^{n} ;\left\|P \cdot \xi^{-1}\right\|<\varepsilon\right\}
$$

for $P \in \mathbf{H}^{n}, \varepsilon>0$. For $0<s<t<2$, define

$$
\begin{aligned}
& U_{i}(s, t)=\left\{\xi \in U_{i} ; s<\left\|\psi_{i}(\xi)\right\|<t\right\}, \\
& U_{i}(t)=\left\{\xi \in U_{i} ;\left\|\psi_{i}(\xi)\right\|<t\right\},
\end{aligned}
$$

$i=1,2$. For $t \in(0,1), A \in U(n)$, we can form a new spherical CR manifold $M_{t, A}$ by removing the closed balls $\overline{U_{i}(t)}, i=1,2$, and identifying $U_{1}(t, 1)$ with $U_{2}(t, 1)$ along the mapping $\Psi_{t, A}: U_{1}(t, 1) \longrightarrow U_{2}(t, 1)$ defined by

$$
\Psi_{2} \circ \Psi_{t, A}(\xi)=\delta_{t} \circ R \circ U_{A}\left(\Psi_{1}(\xi)\right) .
$$


Here $R$ is the inversion defined by $(2.45)$ and $U_{A}$ is the unitary transformation defined by (2.44). Let

$$
\pi_{t, A}: M \backslash \overline{U_{1}(t) \cup U_{2}(t)} \longrightarrow M_{t, A}
$$

be a canonical projection. Since $\Psi_{t, A}$ is CR with respect to the standard CR structure $J$, there is a unique spherical CR structure on $M_{t, A}$, whose pull back along $\pi_{t, A}$ agrees with the structure $J$ restricted to $M \backslash \overline{U_{1}(t) \cup U_{2}(t)}$. We denote this spherical CR structure by $J_{t, A} \cdot\left(M_{t, A}, J_{t, A}\right)$ degenerates to the spherical CR manifold $\left(M \backslash\left\{\xi_{1}, \xi_{2}\right\}, J\right)$ with two punctures $\xi_{1}, \xi_{2}$ as $t \longrightarrow 0$.

Example. Let $\left\{C_{i}, C_{i}^{\prime}\right\}_{i=1}^{k}$ be the boundary of balls $\left\{D_{i}, D_{i}\right\}_{i=1}^{k}$, where $D_{i}$ and $D_{i}^{\prime}$ are mutually disjoint, $D_{i}=B\left(P_{i}, r_{i}\right), D_{i}^{\prime}=B\left(P_{i}^{\prime}, r_{i}^{\prime}\right), P_{i}, P_{i}^{\prime} \in \mathbf{S}^{2 n+1}, r_{i}>0, r_{i}^{\prime}>0$, $i=1, \cdots, k$. Suppose $\gamma_{i}\left(D_{i}\right)=D_{i}^{\prime}, \gamma_{i} \in P U(n+1,1)$. Then, $\left\{\gamma_{i}\right\}$ generates a convex cocompact group $\Gamma$ which is isomorphic to the free group of rank $k$. As in the Riemannian case, we call such group the Schottky group. It is easy to see that $\mathbf{S}^{2 n+1} \backslash \cup_{i=1}^{k}\left(D_{i} \cup D_{i}^{\prime}\right)$ is the fundamental domain for $\Gamma$ [EMM]. $\Omega(\Gamma) / \Gamma$ is CR diffeomorphic to $k\left(\mathbf{S}^{1} \times \mathbf{S}^{2 n}\right)$. Here

$$
k\left(\mathbf{S}^{1} \times \mathbf{S}^{2 n}\right)=\mathbf{S}^{1} \times \mathbf{S}^{2 n_{\sharp}} \cdots \sharp \mathbf{S}^{1} \times \mathbf{S}^{2 n},
$$

$\sharp$ is the connected operation defined by (5.4). By the following proposition, $k\left(\mathbf{S}^{1} \times \mathbf{S}^{2 n}\right)$ admits a spherical CR structure with positive Webster scalar curvature.

Proposition 5.1. If $t$ is sufficiently small, $\left(M_{t, A}, J_{t, A}\right)$ is scalar positive.

Recall the Yamabe invariant

$$
\mu(M, J)=\inf _{f>0} \frac{b_{n} \int_{M}\left|\nabla_{\theta} f\right|^{2} \theta \wedge(d \theta)^{n}+\int_{M} R_{\theta} f^{2} \theta \wedge(d \theta)^{n}}{\left(\int_{M} f^{\frac{2 Q}{Q-2}} \theta \wedge(d \theta)^{n}\right)^{\frac{Q-2}{Q}}},
$$

where $\theta \in\{J\}$. By the solution of the Yamabe problem for connected strictly pseudoconvex CR manifolds (cf. [JL1]-[JL3], [G] and [GY]), there exists $f \in$ $C^{\infty}(M)$ such that the Webster scalar curvature of $f^{\frac{4}{Q-2}} \theta$ is the constant $\mu(M, J)$. Thus, $(M, J)$ is scalar positive if and only if $\mu(M, J)$ is positive. So, Proposition 5.1 follows from the following proposition.

Proposition 5.2. If $t$ is sufficiently small, $\mu\left(M_{t, A}, J_{t, A}\right)>0$.

Proof. The proof is similar to the Riemannian case [Ko]. Let

$$
M_{0}=M \backslash\left\{\xi_{1}, \xi_{2}\right\} .
$$

Choose a contact form $\hat{\theta} \in\{J\}$. Then, by multiplying a positive function $\lambda \in$ $C^{\infty}\left(M_{0} \backslash\left\{\xi_{1}, \xi_{2}\right\}\right)$, we can assume $\theta=\lambda \hat{\theta}$ satisfying

$$
\psi_{i *} \theta(\xi)=\|\xi\|^{-2} \theta_{\mathbf{H}}(\xi) \quad \text { on }(B(0,2)) \backslash\{0\} .
$$

This means that $\left(M_{0}, \theta\right)$ has cylindrical ends. It is easy to see that the gluing mapping $\Psi_{t, A}$ preserves the contact form $\|\xi\|^{-2} \theta_{\mathbf{H}}(\xi)$ on $t<\|\xi\|<\frac{1}{t}, 0<\frac{2}{3} t<1$, by the 
transformation formula (3.18). Hence we can glue $\theta$ along $\Psi_{t, A}$ to obtain a spherical contact form $\theta_{t, A}$ on $M_{t, A}$ satisfying

$$
\pi_{t, A}^{*} \theta_{t, A}=\theta \quad \text { on } \quad M \backslash \overline{U_{1}(t) \cup U_{2}(t)} .
$$

Define a mapping

$$
\Phi: B(0,1) \longrightarrow[0, \infty) \times \Sigma^{n}
$$

by $\Phi(\xi)=\left(\log \frac{1}{\|\xi\|}, \frac{\xi}{\|\xi\|}\right)$, where $\Sigma^{n}=\left\{\xi \in \mathbf{H}^{n} ;\|\xi\|=1\right\}$. Define a contact form $\tilde{\theta}=\Phi_{*}\left(\|\xi\|^{-2} \theta_{\mathbf{H}}\right) .\left(B(0,1),\|\xi\|^{-2} \theta_{\mathbf{H}}\right)$ is CR equivalent to $\left([0, \infty) \times \Sigma^{n}, \tilde{\theta}\right)$. Since

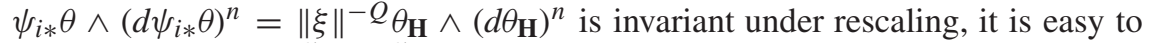
see that the measure $\tilde{\theta} \wedge(d \tilde{\theta})^{n}$ is invariant under translation $(s, x) \longrightarrow\left(s+s_{0}, x\right)$ on $[0, \infty) \times \Sigma^{n}$. As a measure,

$$
\tilde{\theta} \wedge(d \tilde{\theta})^{n}=d l d S_{\Sigma^{n}},
$$

where $d S_{\Sigma^{n}}$ is a measure on $\Sigma^{n}$. We write

$$
\left(M_{0}, \theta\right)=\left([0, \infty) \times \Sigma^{n}, \tilde{\theta}\right) \cup(\hat{M}, \theta) \cup\left([0, \infty) \times \Sigma^{n}, \tilde{\theta}\right),
$$

where $\hat{M}=M \backslash U_{1}(1) \cup U_{2}(1)$. We can glue $(\hat{M}, \theta)$ and $\left([0, l] \times \Sigma^{n}, \tilde{\theta}\right)$ along their boundaries to get $\left(M_{t, A}, J_{t, A}\right)$, where $l=\log \frac{1}{t}$.

By the definition of $\mu\left(M_{t, A}, J_{t, A}\right)$, we can find a positive function $f_{l} \in$ $C^{\infty}\left(M_{t, A}\right)$ such that

$$
\begin{aligned}
b_{n} \int_{M_{t, A}}\left|\nabla_{\theta_{t, A}} f_{l}\right|^{2} \theta_{t, A} \wedge\left(d \theta_{t, A}\right)^{n} & +\int_{M_{t, A}} R_{\theta_{t, A}} f_{l}^{2} \theta_{t, A} \wedge\left(d \theta_{t, A}\right)^{n} \\
& <\mu\left(M_{t, A}, J_{t, A}\right)+\frac{1}{l}
\end{aligned}
$$

and

$$
\int_{M_{t, A}} f_{l}^{\frac{2 Q}{Q-2}} \theta_{t, A} \wedge\left(d \theta_{t, A}\right)^{n}=1 .
$$

By the property of measure $\tilde{\theta} \wedge(d \tilde{\theta})^{n}$ in (5.12), we can show the following claim exactly as Lemma 6.2 in [Ko]: there exists a section $l_{*} \times \Sigma^{n}$ with $0 \leq l_{*} \leq l$ such that

$$
\int_{l_{*} \times \Sigma^{n}}\left(b_{n}\left|\nabla_{\tilde{\theta}} f_{l}\right|^{2}+R_{\tilde{\theta}} f_{l}^{2}\right) d S_{\Sigma^{n}}<\frac{C}{l},
$$

where $C$ is a constant independent of $l$. Now define a Lipschitz function $F_{l}$ on $M_{0}$ by $F_{l}=f_{l}$ on $\left[0, l_{*}\right) \times \Sigma^{n} \cup \hat{M} \cup\left[0, l-l_{*}\right) \times \Sigma^{n}$ and

$$
F_{l}(s, x)=\left\{\begin{array}{lll}
\left(l_{*}+1-s\right) f_{l}\left(l_{*}, x\right) & \text { for } & (s, x) \in\left[l_{*}, l_{*}+1\right] \times \Sigma^{n} \\
0 & \text { for } & (s, x) \in\left[l_{*}+1, \infty\right) \times \Sigma^{n}
\end{array}\right.
$$


and similarly on $\left[l-l_{*}, \infty\right) \times \Sigma^{n}$. By (5.14) and (5.16), we have

$$
b_{n} \int_{M_{0}}\left|\nabla_{\theta} F_{l}\right|^{2} \theta \wedge(d \theta)^{n}+\int_{M_{0}} R_{\theta} F_{l}^{2} \theta \wedge(d \theta)^{n}<\mu\left(M_{t, A}, J_{t, A}\right)+\frac{B}{l},
$$

where $B$ is a constant independent of $l$. Obviously,

$$
\int_{M_{0}} F_{l}^{\frac{2 Q}{Q-2}} \theta \wedge(d \theta)^{n}>1
$$

Therefore,

$$
\inf _{F>0} \frac{b_{n} \int_{M_{0}}\left|\nabla_{\theta} F\right|^{2} \theta \wedge(d \theta)^{n}+\int_{M_{0}} R_{\theta} f^{2} \theta \wedge(d \theta)^{n}}{\left(\int_{M_{0}} F^{\frac{2 Q}{Q-2}} \theta \wedge(d \theta)^{n}\right)^{\frac{Q-2}{Q}}}<\mu\left(M_{t, A}, J_{t, A}\right)+\frac{B}{l}
$$

where the infimum is taken over all nonnegative Lipshitz functions with compact support. Since for compact supported function $f$, the integrals in the left side of (5.20) is equal to integrals on $M$ with respect to $\theta^{*}$ with $\theta^{*}=\theta$ on $\operatorname{supp} f$. Thus, it follows from the definition of the Yamabe invariant that the left side is greater than or equal to $\mu(M, J)$. If $(M, J)$ is a disjoint union of $\left(M_{1}, J_{1}\right)$ and $\left(M_{2}, J_{2}\right)$, we have

$$
\mu(M, J)=\min \left\{\mu\left(M_{1}, J_{1}\right), \mu\left(M_{2}, J_{2}\right)\right\},
$$

by the definition of Yamabe invariant. From (5.20) and (5.21), $\mu\left(M_{t, A}, J_{t, A}\right)$ is positive if $l$ is sufficiently large, i.e. $t$ is sufficiently small. The proposition is proved.

Remark 5.3. For radial function $u$, i.e. $u(\xi)=f(\|\xi\|)$ for some function $f$ on $\mathbf{R}_{+}$, we have (cf. [GL, p. 327],

$$
\triangle_{\theta_{\mathbf{H}}} u(\xi)=-\psi_{0}(\xi)\left[f^{\prime \prime}(\|\xi\|)+\frac{Q-1}{\|\xi\|} f^{\prime}(\|\xi\|)\right],
$$

where $\psi_{0}(\xi)=\frac{|z|^{2}}{\|(z, t)\|^{2}}$ for $\xi=(z, t) \neq 0$. It is easy to see that the Webster scalar curvature of $\|\xi\|^{-2} \theta_{\mathbf{H}}$ is non-negative and vanishes on the axis $\left\{(z, t) \in \mathbf{H}^{n} \mid z=0\right\}$.

\section{References}

[A] Aubin, T.: Nonlinear analysis on manifolds: Monge-Ampère equations. Grundlehren Math. Wiss. 252. Berlin: Springer 1982

[BFG] Beals, M., Fefferman, C., Grossman, R.: Strictly pseudoconvex domains in $\mathbf{C}^{n}$. Bull. Am. Math. Soc., New Ser. 8, 125-322 (1983)

[BG] Beals, R., Greiner, P.: Calculus on Heisenberg manifolds. Ann. Math. Stud. 119. Princeton, NJ: Princeton University Press 1988

[BS] Burns, D., Shnider, S.: Spherical hypersurfaces in complex manifolds. Invent. Math. 33, 223-246 (1976)

[CL] Cheng, J.-H., Lee, J.M.: A local slice theorem for 3-dimensional CR structures. Am. J. Math. 117, 1249-1298 (1995)

[CM] Chern, S.S., Moser, J.: Real hypersurfaces in complex manifolds. Acta Math. 133, 219-271 (1974) 
[C] Corlette, K.: Hausdorff dimension of limit sets I. Invent. Math. 102, 521-541 (1990)

[EMM] Epstein, C.L., Melrose, R.B., Mendoza, G.A.: Resolvent of the Laplacian on strictly pseudoconvex domains. Acta Math. 167, 1-106 (1991)

[G] Gamara, N.: CR Yamabe conjecture-the conformally flat case. Pac. J. Math. 201, 121-175 (2001)

[GY] Gamara, N., Yacoub, R.: The CR Yamabe conjecture the case $n=1$. J. Eur. Math. Soc. (JEMS) 3, 105-137 (2001)

[GL] Garofalo, N., Lanconelli, E.: Frequency functions on the Heisenberg group, the uncertainty principle and unique continuation. Ann. Inst. Fourier 40, 313-356 (1990)

[GT] Gilbarg, D., Trudinger, N.S.: Elliptic partial differential equations of second order. Grundlehren Math. Wiss. 224. Berlin: Springer 1977

[Gr] Gray, J.W.: Some global properties of contact structures. Ann. Math. (2) 69, 421-450 (1959)

[H] Habermann, L.: Riemannian metrics of constant mass and moduli spaces of conformal structures. Lect. Notes Math. 1743. Berlin: Springer 2000

[HJ] Habermann, L., Jost, J.: Green functions and conformal geometry. J. Differ. Geom. 53, 405-442 (1999)

[I] Izeki, H.: Limits sets of Kleinian groups and conformally flat Riemannian manifolds. Invent. Math. 122, 603-625 (1995)

[JL1] Jerison, D.S., Lee, J.M.: The Yamabe problem on CR manifolds. J. Differ. Geom. 25, 167-197 (1987)

[JL2] Jerison, D.S., Lee, J.M.: Extremals for the Sobolev inequality on the Heisenberg group and the CR Yamabe problem. J. Am. Math. Soc. 1, 1-13 (1988)

[JL3] Jerison, D.S., Lee, J.M.: Intrinsic CR coordinates and the CR Yamabe problem. J. Differ. Geom. 29, 303-343 (1989)

[KT] Kamishima, Y., Tsuboi, T.: CR-structures on Seifert manifolds. Invent. Math. 104, 149-163 (1991)

[Ko] Kobayashi, O.: Scalar curvature of a metric with unit volume. Math. Ann. 279, 253-265 (1987)

[K] Kulkarni, R.S.: On the principle of uniformization. J. Differ. Geom. 13, 109-138 (1978)

[KP] Kulkarni, R.S., Pinkall, U.: A canonical metric for Möbius structures and its applications. Math. Z. 216, 89-129 (1994)

[Le] Lee, J.M.: The Fefferman metric and pseudo-Hermitian invariants. Trans. Am. Math. Soc. 296, 411-429 (1986)

[L] Leutwiler, H.: A Riemannian metric invariant under Möbius transformations in $\mathbf{R}^{n}$. Lect. Notes Math. 1351, pp. 223-235. Berlin: Springer 1988

[Li1] Li, Z.: Uniformization of spherical CR manifolds and the CR Yamabe problem. Proc. Symp. Pure Math. 54, Part 1, 299-305 (1993)

[Li2] Li, Z.: Hausdorff dimension of limit sets for spherical CR manifolds. J. Funct. Anal. 139, 1-28 (1996)

[N] Nayatani, S.: Patterson-Sullivan measure and conformally flat metrics. Math. Z. 225, 115-131 (1997)

[SY] Schoen, R., Yau, S.-T.: Conformally flat manifolds, Kleinian groups and scalar curvature. Invent. Math. 92, 47-71 (1988)

[Wa] Wang, W.: Positive solution of a subelliptic nonlinear equation on the Heisenberg group. Can. Math. Bull. 44, 346-354 (2001)

[W] Webster, S.M.: Pseudo-Hermitian structures on a real hypersurface. J. Differ. Geom. 13, 25-41 (1978) 